\title{
REVISTAACIÓN
}

Revista Educación

ISSN: 0379-7082

ISSN: 2215-2644

revedu@gmail.com

Universidad de Costa Rica

Costa Rica

\section{Relato de una experiencia de promoción de la salud en escuelas: fomentando el lavado de manos}

Oberto, María Georgina; Mamondi, Verónica; Ferrero, Mercedes; Sánchez, Roberto José

Relato de una experiencia de promoción de la salud en escuelas: fomentando el lavado de manos

Revista Educación, vol. 44, núm. 2, 2020

Universidad de Costa Rica, Costa Rica

Disponible en: http://www.redalyc.org/articulo.oa?id=44062184003

DOI: https://doi.org/10.15517/revedu.v44i2.37581

Esta obra está bajo una Licencia Creative Commons Atribución-NoComercial-SinDerivar 3.0 Internacional. 


\section{Relato de una experiencia de promoción de la salud en escuelas: fomentando el lavado} de manos

\section{A Narrative Account of Health Promotion in Schools: Encouraging Hand Washing}

Maria Georgina Oberto

Universidad Nacional de Córdoba, Argentina

georginaoberto@hotmail.com

(iD http://orcid.org/0000-0002-1921-7926

Verónica Mamondi

Universidad Nacional de Córdoba, Argentina

vmamondi@fcm.unc.edu.ar

(iD) http://orcid.org/0000-0001-9074-6014

Mercedes Ferrero

Universidad Nacional de Córdoba, Argentina

ferreromercedes@yahoo.com.ar

iD http://orcid.org/0000-0001-9958-7267

Roberto José Sánchez

Universidad Nacional de Córdoba, Argentina

rosan60@hotmail.com

iD http://orcid.org/0000-0002-9323-5989
DOI: https://doi.org/10.15517/revedu.v44i2.37581

Redalyc: http://www.redalyc.org/articulo.oa?id=44062184003
Recepción: 02 Julio 2019

Aprobación: 01 Febrero 2020

\section{Resumen:}

La promoción de la salud en el entorno escolar, con estrategias y recursos didácticos adecuados, propicia el desarrollo de comportamientos favorecedores para la salud. Así, los objetivos fueron promover el correcto lavado de manos como método de prevención de enfermedades de transmisión alimentaria y de otras enfermedades diarreicas y respiratorias, para fortalecer prácticas de higiene cotidianas en la población escolar de dos escuelas de nivel inicial y primario, de la ciudad de Córdoba, en el periodo 2016-2018. También, elaborar materiales educativos sobre la temática. Las actividades de promoción de la salud fueron llevadas a cabo por cinco docentes y diez estudiantes de la Universidad Nacional de Córdoba, Argentina. En cada año, el diagnóstico de situación, permitió conocer los equipos docentes y acordar los contenidos por trabajar al tener en cuenta el currículo escolar. Se organizaron talleres educativos con trabajos prácticos en los sanitarios para el correcto lavado de manos. Entre 2016-2018 participaron 504 estudiantes y 20 maestras de ambas escuelas. Los talleres dieron como resultado un libro infantil, afiches de difusión y 32 producciones infantiles de material artístico-didáctico exhibidas en la Noche de los Museos, 2018. La mayoría de las maestras consideraron la intervención adecuada y constructiva. Las niñas con respecto a los niños, evidenciaron una mejor adquisición de la práctica de lavado de manos luego de usar los sanitarios, siendo insuficiente el estudiantado que incorporó el hábito higiénico. Se concluye que la escuela es un entorno favorable para promover comportamientos saludables, y es necesario continuar fortaleciendo las prácticas.

Palabras Clave: Educación sanitaria, Lavado de manos, Higiene, Escuela, Salud infantil.

\section{Abstract:}

Encouraging healthy habits in schools through appropriate teaching strategies and resources promotes good health. The objectives of this study are to instill correct hand washing techniques as a method of preventing foodborne illnesses and other diarrheal and respiratory diseases, strengthen daily hygiene practices at two schools in Cordoba, Argentina during a two-year period of 2016-2018, specifically among pre-school and primary school levels, and, finally, develop educational materials on hand washing. Health promotion activities were conducted by five instructors and ten students from the National University of Córdoba. Each 
year, an assessment of the situation was made to introduce the teaching teams and agree on how the information would be presented while taking the school curriculum into account. Educational workshops were carried out with practical work in school restrooms to teach proper handwashing. During the 2016 and 2018 period, 504 students and 20 teachers from both schools attended the workshops. The outputs resulted in a children's book, dissemination posters and 32 examples of original artwork by children to be used as educational material which was exhibited at an event called, Night of the Museums in 2018. The workshops and outputs were readily accepted and considered appropriate by the school teachers. As a result, girls displayed better assimilation of hand washing after using the toilet more than boys. Yet, the number of students that acquired the habit was lower than expected. It was concluded, however, that schools are favorable environments to encourage and promote healthy behaviors, but that more work is needed to continue to strengthen this practice.

KEYworDs: Health Education, Hand Washing, Handwashing, Hygiene, School, Child Health.

\section{INTRODUCCIÓN}

La Organización Mundial de la Salud [OMS] en su definición de salud destaca el valor de la prevención al incluir los aspectos físico, mental y social y no solo la ausencia de afecciones o enfermedades. Si bien, esta concepción de salud ha recibido críticas durante años, no ha sido enmendada desde 1948. Posteriormente, los contenidos de la Carta de Ottawa y los subsiguientes acuerdos internacionales reafirman la importancia de la promoción de la salud para mejorar la calidad de vida y la equidad sanitaria (OMS, 1986). En consecuencia, una de las funciones de la promoción de la salud es fomentar el desarrollo de conocimientos, capacidades y aptitudes para que las personas puedan tener mayor control de su salud e incorporen hábitos saludables que reduzcan las conductas de riesgo (Ippolito y Mantilla, 2003; OMS, 2016). Dentro de esta concepción la salud escolar promueve la salud de la comunidad educativa, así como el control higiénico de los establecimientos educativos. De esta manera, educación para la salud en el entorno escolar, contribuye al desarrollo de comportamientos psico-sociales favorables (Morón, 2015). Es por ello, que las intervenciones deben implementar estrategias y recursos didácticos basados en los paradigmas actuales en pedagogía, centrados en el estudiantado y su contexto, donde la escuela, ámbito propicio para educar en salud, favorece el aprendizaje de prácticas beneficiosas para la salud que pueden perdurar a lo largo de la vida (Rodríguez et al, 2017; Beltrán, Navarro y Peña, 2018).

El lavado de manos, es una de las maneras más efectiva, sencilla y económica para prevenir enfermedades de transmisión alimentaria [ETA] y otras enfermedades diarreicas y respiratorias, que en forma conjunta son las causantes de alrededor de 3,5 millones de muertes en menores de cinco años (Organización Panamericana de la Salud [OPS], 2011; Fondo de las Naciones Unidas para la Infancia [UNICEF], 2018). Estudios efectuados en la población infantil dan cuenta de que se conoce la importancia del lavado de manos en el entorno familiar, pero existen debilidades en las personas cuidadoras sobre los pasos para el correcto lavado de manos (Soto, Gómez, Parrado, Hernández y Gómez, 2017). Del mismo modo, la mayoría del estudiantado, en la escuela y en el hogar, no ejecuta una práctica correcta del lavado de manos, tanto en su procedimiento, como en sus momentos, y las Instituciones educativas no cuentan con los elementos suficientes para una práctica adecuada (Caballero, Flores, Bonilla y Arenas, 2017; Soto et al., 2017).

Además, la higiene de manos cumple un rol fundamental en la reducción de infecciones intrahospitalarias y en el hogar (Luby, Halder, Huda, Unicomb y Johnston, 2011; Haverstick et al, 2017), y a pesar de ser una barrera protectora que contribuye a reducir un $25 \%$ las infecciones respiratorias y un $40 \%$ las diarreas infantiles, rara vez se practica en los momentos críticos, como antes de comer y después de ir al baño, y no siempre resulta fácil promoverla (OPS, 2011; UNICEF, 2018). En ese sentido, la Global Handwashing Partnership (2017) celebra cada 15 de octubre el Día Mundial del Lavado de Manos con el propósito de reforzar los programas de promoción de la higiene y saneamiento que desarrollaron los países. Es un gran desafío enraizar la práctica habitual de esta norma elemental de aseo, siendo clave en la estrategia de entornos escolares saludables, en la cual se necesita un comportamiento interiorizado y consciente en los hogares y en las escuelas, que imprime desafíos y retos cuando se educa en salud (Herrero, López y Martínez, 2017). 
La escuela, que es donde se congrega un gran número de niños y niñas, es un espacio propicio para la propagación de infecciones respiratorias y gastrointestinales, que por lo general son autolimitadas, pero altamente contagiosas pudiendo afectar a comunidades escolares enteras, y extenderse en el hogar a grupos más vulnerables, como hermanas y hermanos menores (Willmott et al., 2015). Esto lleva a oportunidades pedagógicas perdidas, las cuales pueden tener un efecto perjudicial en los resultados educativos, pérdida de productividad y de días de trabajo en el personal docente. No obstante, la cooperación entre la Universidad y otros actores de la sociedad, puede contribuir a mejorar la calidad de vida de las personas, desde un enfoque interactivo y dialógico entre los conocimientos científicos y las necesidades de la comunidad que participa (Red Nacional de Extensión Universitaria [REXUNI], 2012). Intervenciones sanitarias en escuelas, reportan que el estudiantado mejora el cumplimiento del lavado de manos en general y en particular tras usar los sanitarios (Ejemot, Ehiri, Arikpo, Meremikwu y Critchley, 2015; Caballero et al., 2017; Wichaidit et al., 2019).

Frente a estos antecedentes, y entendiendo que el ámbito escolar junto a la familia son la principal fuente generadora de conocimiento en los niños y las niñas, siendo un período potencial para adquirir hábitos positivos en salud que los acompañarán en su vida adulta, un grupo de cinco docentes de la Facultad de Ciencias Médicas [FCM] de la Universidad Nacional de Córdoba [UNC], Argentina (cátedras de Microbiología y Parasitología y Ciencias Psicosociales de la carrera de Licenciatura en Nutrición), conformaron un equipo interdisciplinario (dos nutricionistas, un médico, una bioquímica y una psicóloga) junto a diez estudiantes universitarios que cursaban las asignaturas.

El trabajo se centró en impulsar una difusión masiva y sostenida en el tiempo de la práctica correcta del lavado de manos, a fin de que las intervenciones para mejorar la higiene de manos en los centros educativos, puedan contribuir a reducir la incidencia de las ETA e infecciones respiratorias y gastrointestinales en escolares, que a su vez, permiten a largo plazo preservar salud integral de toda la comunidad educativa.

Las actividades se organizaron en dos Instituciones de nivel inicial y primario, pública y privada, de la ciudad de Córdoba, Argentina, en el marco de un proyecto de extensión universitaria aprobado por Resoluciones del Honorable Consejo Directivo N 211 (2017), 1450 (2017) y 859 (2018) de la FCMUNC. El objetivo fue promover el correcto lavado de manos como método de prevención de ETA y de otras enfermedades diarreicas y respiratorias, para fortalecer prácticas de higiene cotidianas en la población escolar de dos escuelas de nivel inicial y primario, de la ciudad de Córdoba, en el periodo 2016-2018. Además, ante la demanda de las Instituciones de contar con material ilustrativo para el trabajo en las aulas, se propuso fortalecer los hábitos higiénicos cotidianos tendientes a preservar la salud en las y los niños, mediante la elaboración de material bibliográfico (libro infantil y carteles educativos).

\section{Procedimientos metodológicos}

Se llevó a cabo un proyecto de intervención educativa para la promoción de la salud, bajo la postura pedagógica constructivista socio-cognitiva de Vigostky, considerando un enfoque dialéctico social (Martínez y Zea, 2004). Las actividades fueron dirigidas a la población estudiantil de nivel inicial y primer ciclo de educación primaria, docentes y autoridades de la comunidad educativa de un establecimiento de educación pública y otro privado de la zona noroeste de la ciudad de Córdoba, Argentina. Las escuelas fueron seleccionadas por conveniencia, a partir de comunicaciones de miembros del equipo de trabajo con las maestras y directivos de dichos establecimientos interesados en incorporar en sus currículos la temática propuesta. Se contó con el aval Institucional de ambos establecimientos educativos y a través del cuaderno de comunicados, se informó a las personas cuidadoras los alcances de la intervención educativa y se solicitó firma de consentimiento informado.

El proyecto de promoción de la salud se llevó a cabo durante tres años, desarrollando un seguimiento del alumnado y sumando al nuevo matriculado anualmente en las escuelas. Se valoró la infraestructura de los 
sanitarios, la participación del estudiantado en los talleres educativos y la opinión de las docentes sobre la experiencia. Los instrumentos para recolectar los datos se diseñaron para el presente proyecto. Se describieron los datos mediante frecuencias absolutas y relativas utilizando el software Excel para las variables cuantitativas y para las cualitativas se describió su contenido.

El proyecto consideró cinco etapas: 1. Preparación del equipo de trabajo. 2. Contacto y diagnóstico de las Instituciones educativas. 3. Intervención propiamente dicha. 4. Evaluación de la intervención. 5. Producción de materiales educativos.

\section{Preparación del equipo de trabajo}

El equipo docente universitario, luego de varias reuniones, fijó las líneas de acción. Inicialmente, se organizó un taller sanitario educativo con el estudiantado de la universidad a fin de proporcionar información precisa sobre la práctica correcta del lavado de manos y planificar las actividades.

\section{Contacto y diagnóstico de las Instituciones educativas}

En cada año, se ejecutó el diagnóstico de situación con la finalidad de conocer las maestras de nivel inicial y primario, debido a que suele ocurrir movimiento de cargos por reasignaciones a otros grados y/o traslados de interinatos. Desde este punto, se acordaron los contenidos a trabajar teniendo en cuenta el currículo y las características del grupo escolar.

En el año 2016, en la entrevista con las maestras de nivel inicial de la escuela pública se registró que la temática sobre el lavado de manos es abordada a través del proyecto institucional de la buerta y la cosecha de verduras cuando desarrollan la manipulación de verduras y las prácticas de higiene en ese proceso. En ambas escuelas, la práctica del lavado de mano, está formalmente establecida previa a la merienda y la misma es acompañada por las docentes. Sin embargo, en la jornada escolar, no asisten al alumnado en otros momentos a lavarse las manos.

En la primaria, en ambas escuelas, el lavado de manos está contemplado dentro del currículo en la unidad $e l$ cuerpo humano, la salud y la bigiene. Dicha temática se desarrolla como recomendación general y su práctica no es supervisada por las docentes. Esta instancia permitió recuperar de los currículos escolares prácticas y saberes en relación con hábitos de higiene y prevención de enfermedades. Además, se definió el cronograma y la dinámica de los talleres para las salas y grados participantes.

Se aplicó una guía de observación de sanitarios, la cual completaron estudiantes universitarios, en un primer trabajo de campo en las escuelas (Figura 1). Además, se efectuó observación directa en los sanitarios de la escuela, durante una jornada escolar, completando un formulario adaptado de la OMS (2010) con las opciones: no se lavó, se lavó solo con agua, se lavó con agua y jabón; con la finalidad de registrar el número de escolares que se lavó las manos y su práctica. 


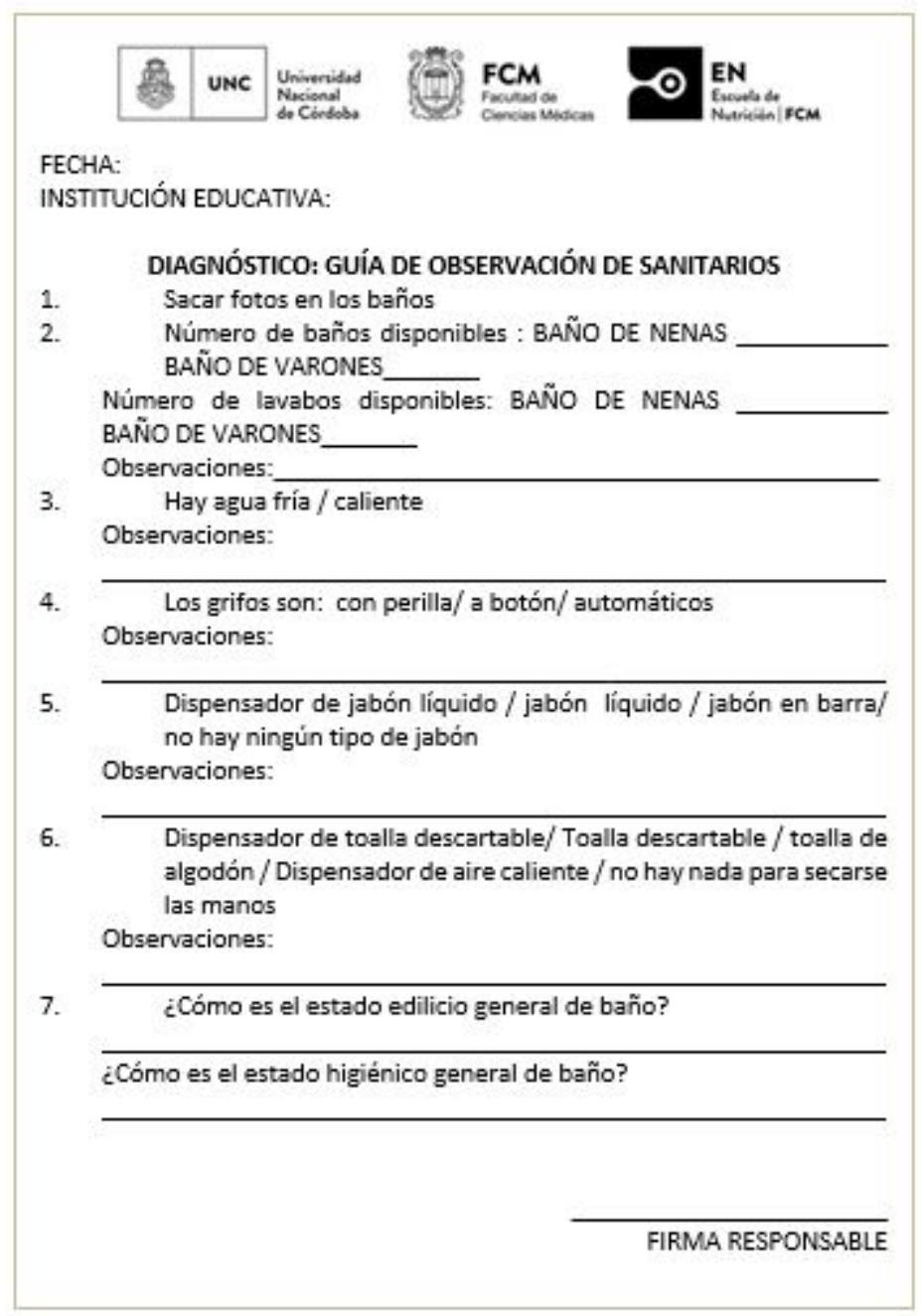

FIGURA 1

Guía de observación de sanitarios

Fuente: Elaboración propia

En el año 2017, se organizaron reuniones con las maestras y directivos de la escuela privada para retomar las actividades (lamentablemente, por razones ajenas a la responsabilidad de este equipo de investigación, no se pudo continuar trabajando con la escuela pública). Se dialogó sobre las acciones que promueve la escuela en relación con la práctica de higiene de manos a partir de la vivencia del trabajo del año previo, y de esta manera recuperar prácticas y saberes del currículo escolar en cuanto a hábitos de higiene y prevención de enfermedades, y fomentar su institucionalización. Con la información recabada se generaron ideas para elaborar las actividades lúdicas sobre la temática.

Se valoró el compromiso institucional en cuanto a la provisión de elementos necesarios para un correcto lavado de manos, derivado de los aportes del trabajo del año anterior, mediante la verificación de papel descartable y jabón líquido en los sanitarios.

En el año 2018 se dio continuidad a las actividades en otro ámbito diferente a las escuelas, con la finalidad de ampliar a las personas destinatarias y compartir las producciones desarrolladas en los años anteriores del proyecto. Se participó en el marco de la Noche de los Museos, actividad cultural nocturna que es organizada anualmente y en forma conjunta por la UNC, la Municipalidad de la ciudad Córdoba y la Agencia Córdoba Cultura del Gobierno de la Provincia (Universidad Nacional de Córdoba [UNC], 2018). 


\section{Intervención propiamente dicha en la institución educativa}

En el año 2016 se efectuaron once talleres educativos con estudiantes y maestras. Cada taller consistió en tres momentos:

1. Momento inicial: se dialogó abiertamente con el estudiantado para conocer qué pensaban y qué hacían habitualmente en relación con la higiene de manos. La información recabada permitió generar ideas para elaborar mensajes educativos sobre la temática y luego diseñar un material bibliográfico para el aula. Este diálogo abierto, se logró utilizando distintos recursos, como actividades lúdicas, presentaciones audiovisuales en PowerPoint, video infantil y canciones (Figura 2) donde se mostraron recomendaciones para un adecuado lavado de manos. Así, mediante el juego, la motivación visual y auditiva se facilitó la apropiación de los conocimientos (Figura 3).

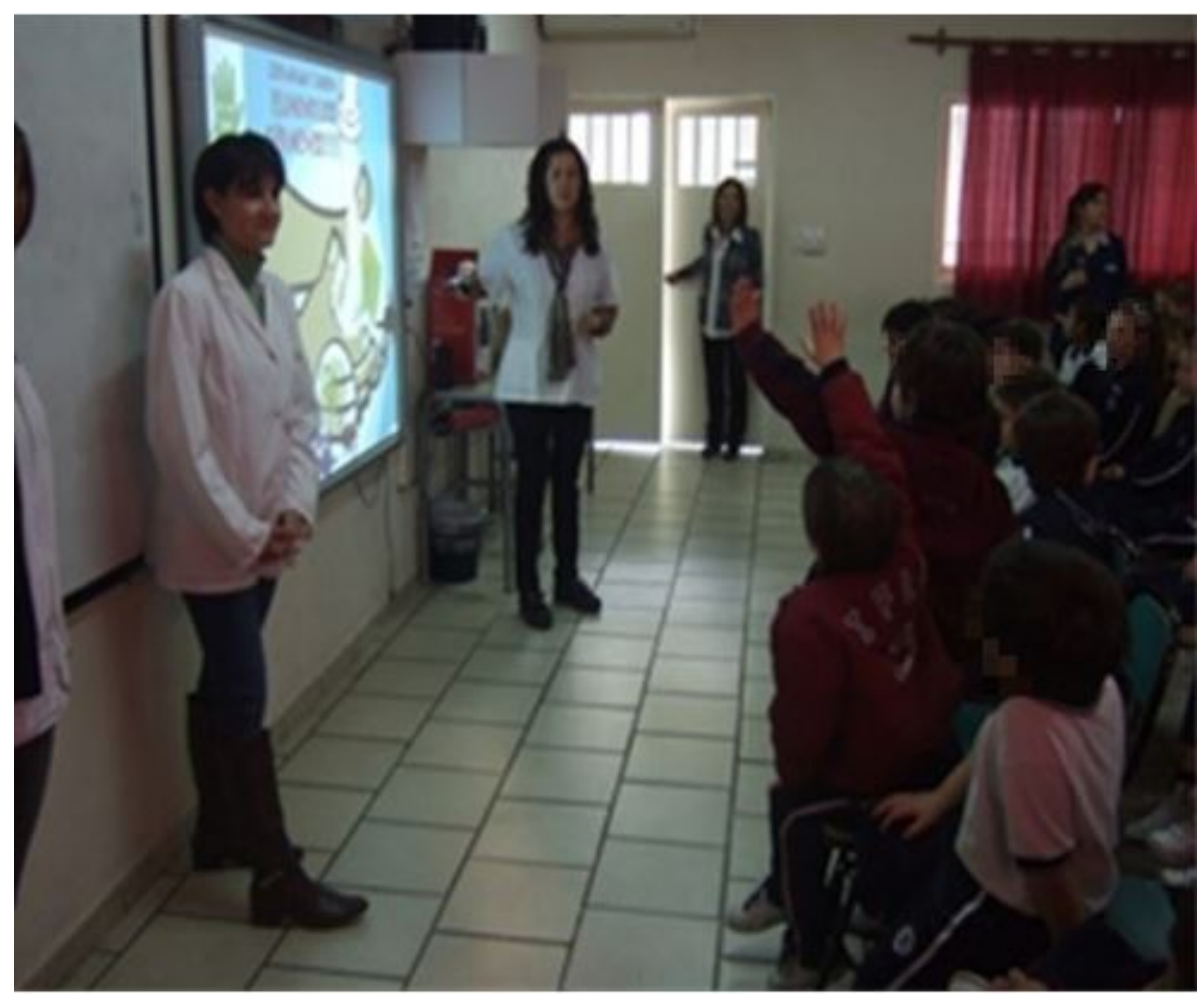

FIGURA 2

Momento inicial. Fotografía de las exposiciones dialogadas en el aula Fuente: Elaboración propia. 


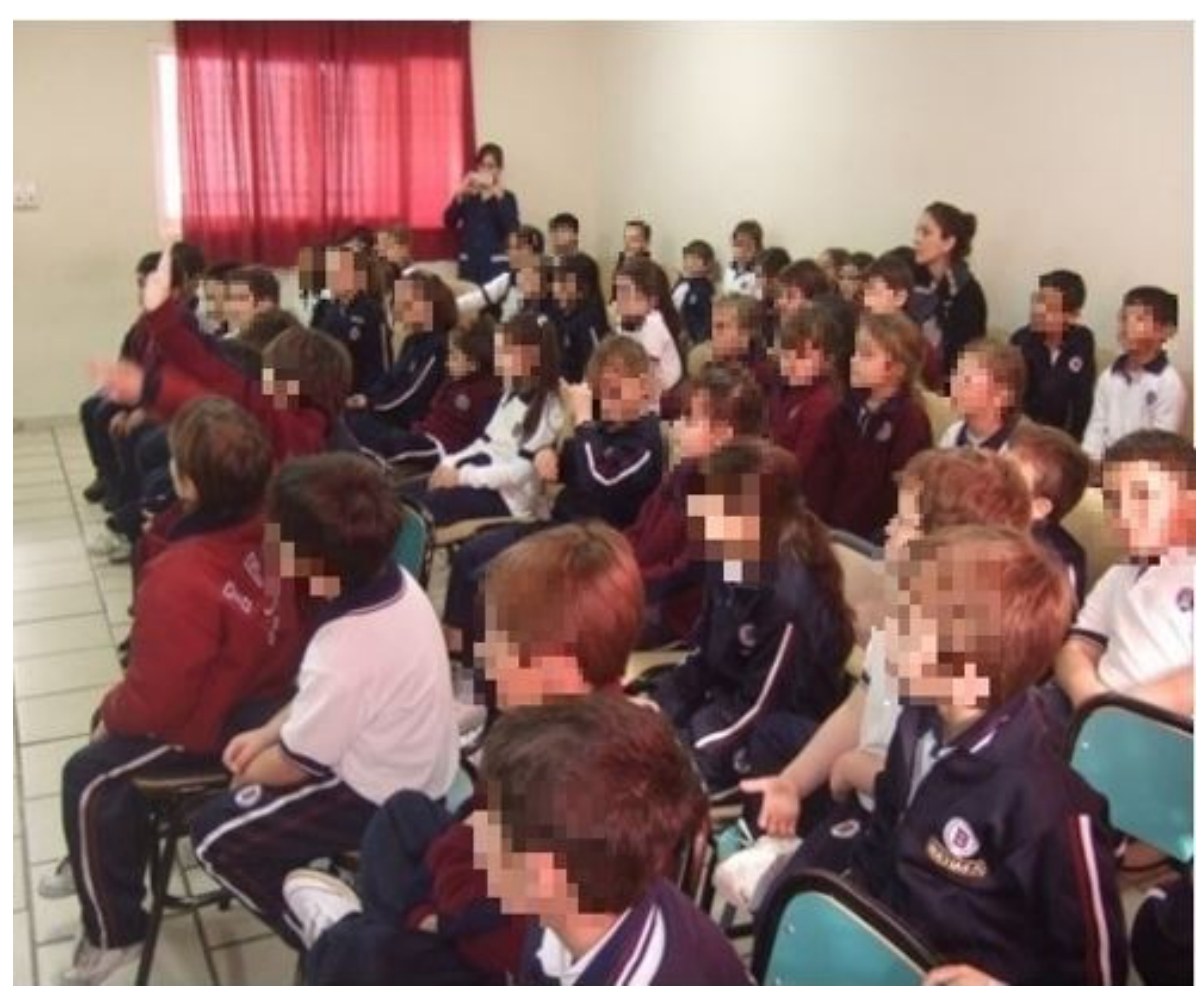

FIGURA 3. MOMENTO INICIAL

Fotografía de estudiantes participando de las exposiciones dialogadas en el aula Fuente: Elaboración propia.

2. Momento de trabajo práctico: en los sanitarios bajo el lema Yo me lavo las manos, ¿y vos? Se hicieron en grupos reducidos, de acuerdo con la capacidad de las instalaciones y lavabos disponibles. La actividad se organizó de modo tal que todas y todos los escolares, acompañados por un integrante del equipo universitario, tuvieron la experiencia de lavarse las manos como parte del proceso que vincula experiencia-reflexión-acción. Se observó la práctica de cada estudiante efectuando las adecuaciones necesarias para el correcto lavado de manos. La experiencia se llevó a cabo tanto en la escuela pública (Figuras 4 y 5) como en la privada (Figura 6). A partir de la práctica y de la reflexión permanente de la realidad, se contribuye a que las y los sujetos tengan elementos para pasar a la acción transformadora, de incluir cotidianamente la higiene de manos. 


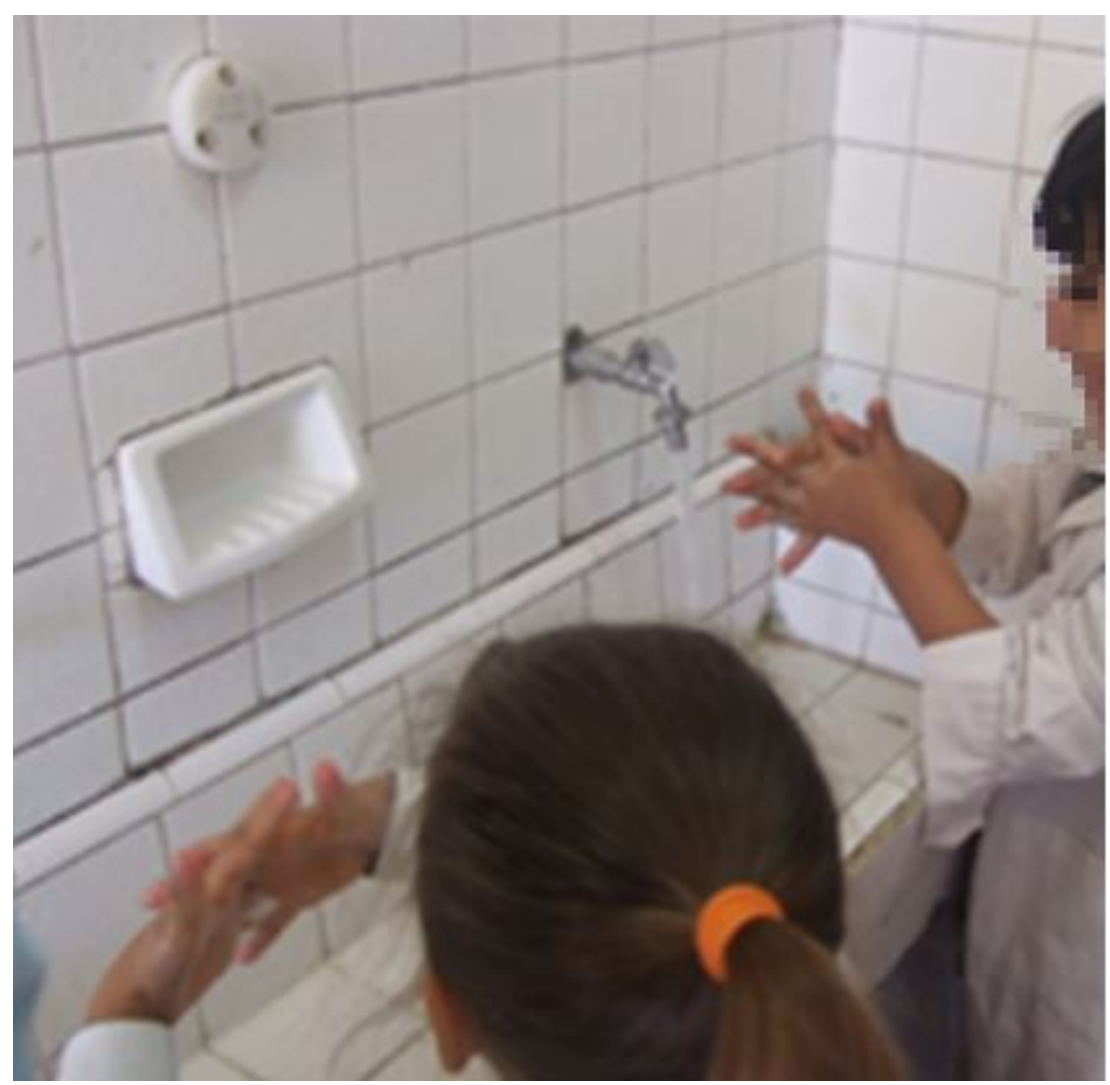

FIGURA 4. MOMENTO DE TRABAJO PRÁCTICO EN LOS SANITARIOS

Fotografía de estudiantes de la escuela pública ensayando la práctica correcta del lavado de manos Fuente: Elaboración propia. 


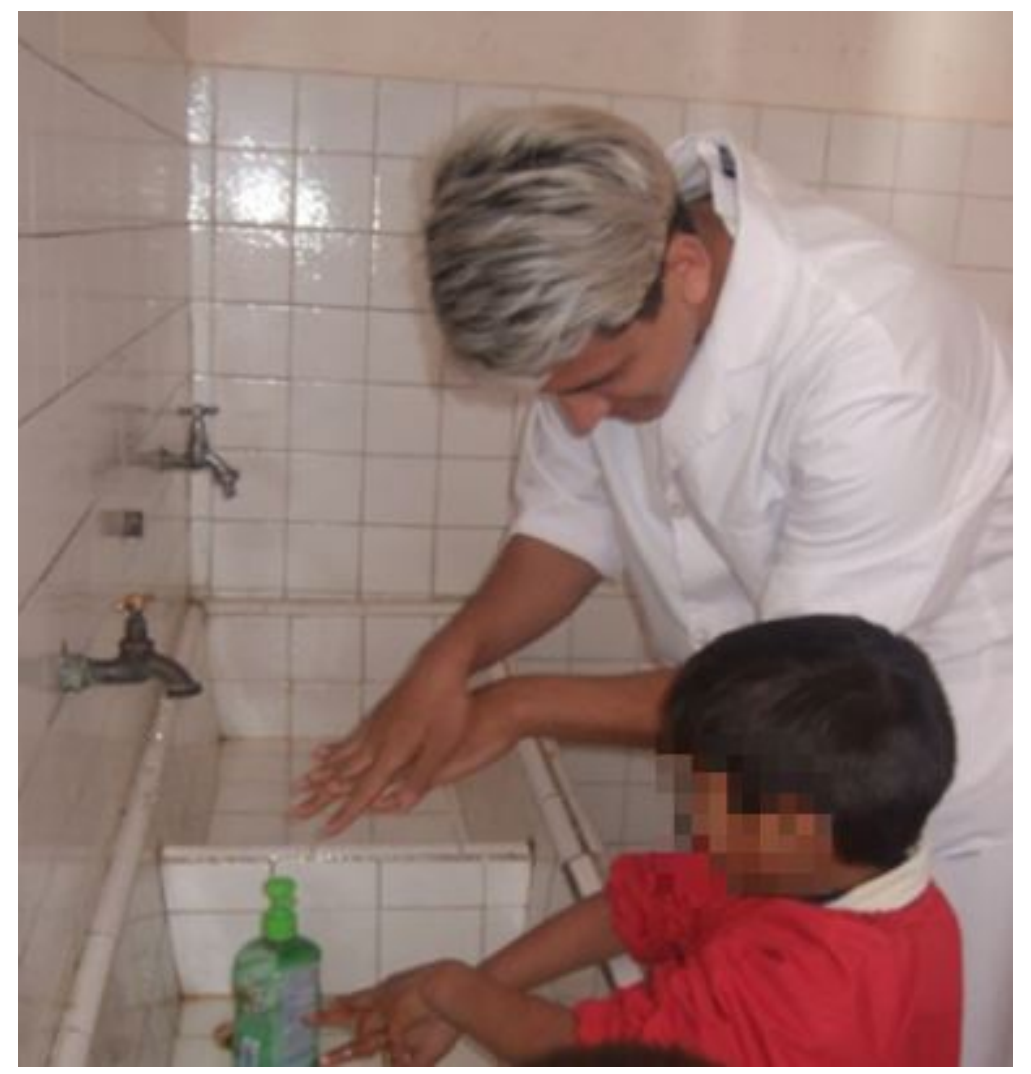

FIGURA 5

Momento de trabajo práctico en los sanitarios. Fotografía de estudiante de la escuela pública ensayando la práctica correcta del lavado de manos. Fuente: Elaboración propia.

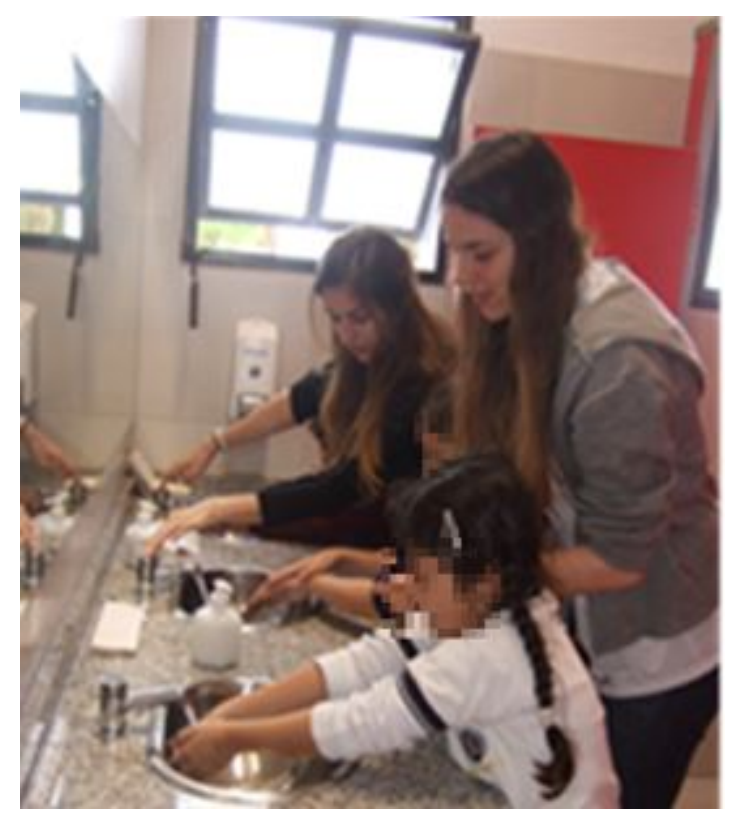

FIGURA 6

Momento de trabajo práctico en los sanitarios. Fotografía de estudiantes de la escuela privada ensayando la práctica correcta del lavado de manos Fuente: Elaboración propia. 
3. Momento de cierre: en las aulas, el alumnado trabajó individualmente en sus cuadernos con actividades didácticas para afianzar lo aprendido sobre la importancia del lavado de manos y su adecuada implementación. Las actividades se complejizaron según la edad de las y los destinatarios.

Durante el año 2017 se llevaron a cabo seis talleres de cuenta cuentos y juegos con alumnos y maestras, utilizando como principal recurso el libro infantil elaborado el año anterior (Oberto, Ferrero, Mamondi, Vaca y Sánchez, 2016) (Figuras 7, 8 y 9).

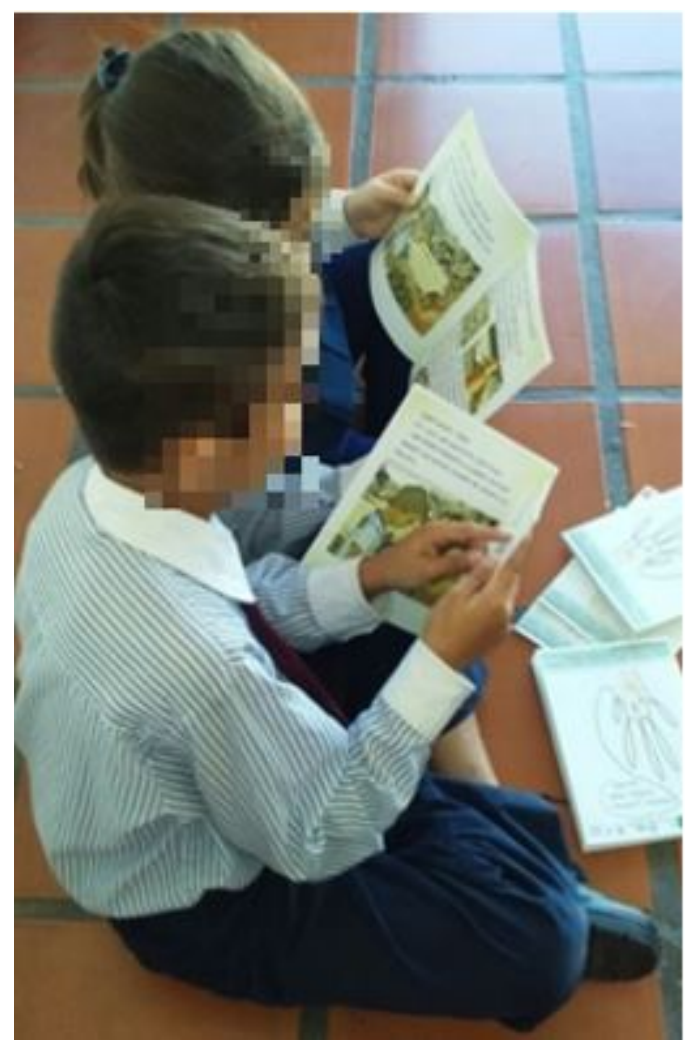

FIGURA 7

Taller cuenta cuentos y juegos. Fotografía de estudiantes de la escuela privada utilizando el libro infantil: Manos limpias. Niños más sanos (Oberto et al., 2016).

Fuente: Elaboración propia. 


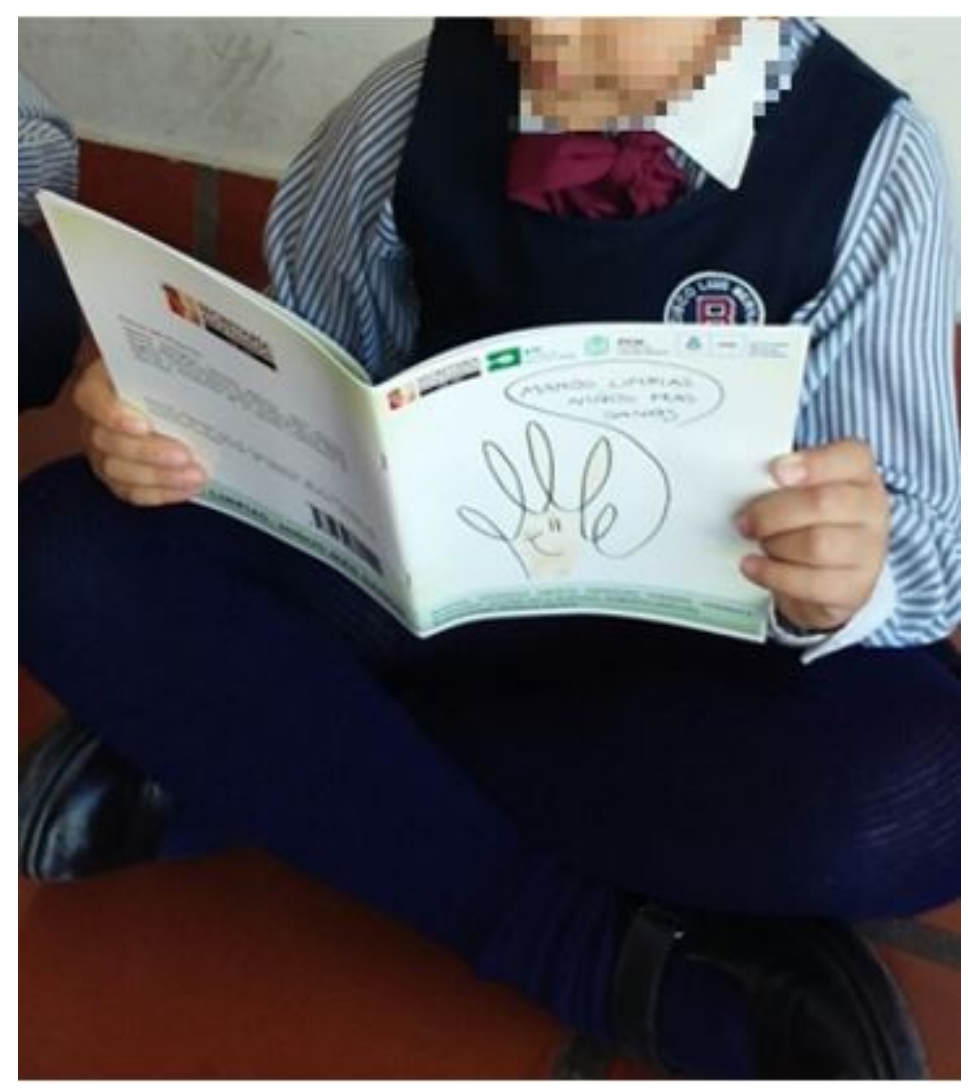

FIGURA 8

Taller cuenta cuentos y juegos. Fotografía de estudiante de la escuela privada utilizando el libro infantil: Manos limpias. Niños más sanos (Oberto et al., 2016). Fuente: Elaboración propia. 


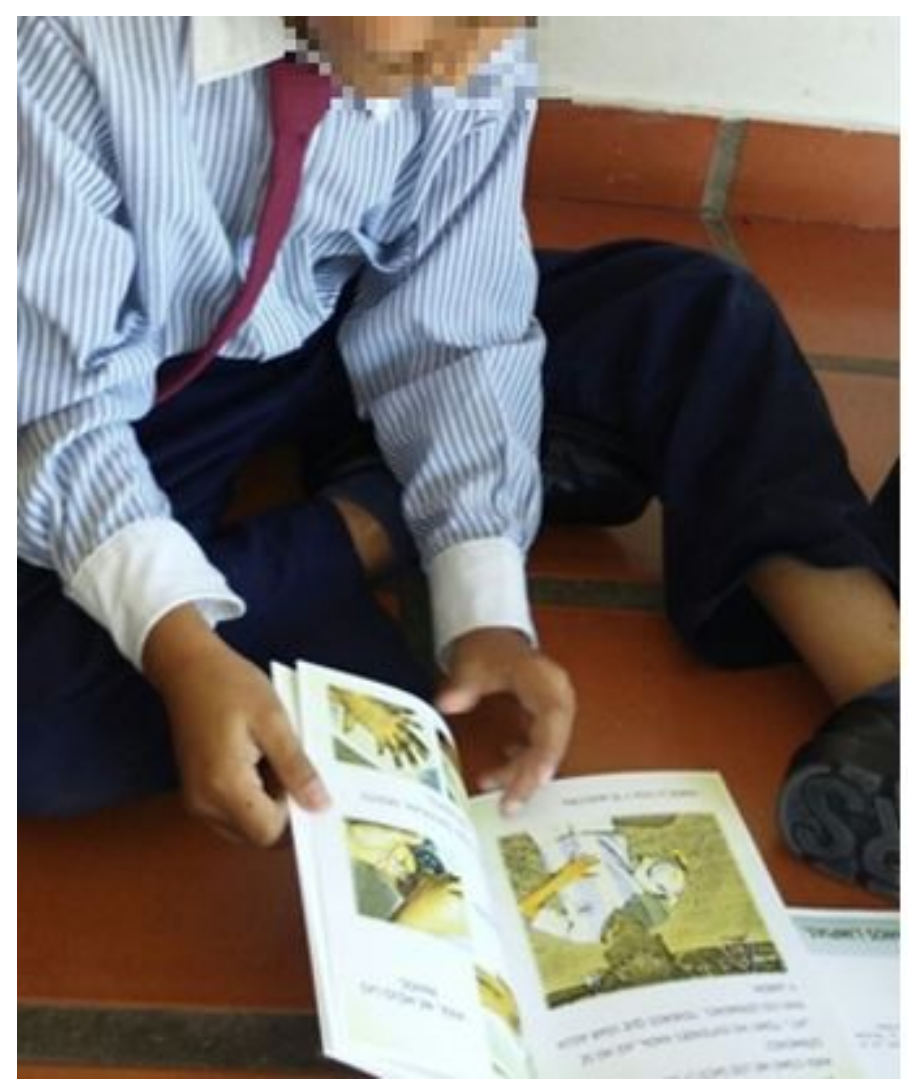

FIGURA 9

Taller cuenta cuentos y juegos. Fotografía de estudiante de la escuela privada utilizando el libro infantil: Manos limpias. Niños más sanos (Oberto et al., 2016).

Fuente: Elaboración propia.

Cada taller contó con los siguientes momentos:

1. Momento inicial: se dialogó con el alumnado para recordar la práctica correcta de higiene de manos. Considerando que las nuevas generaciones de estudiantes están cada vez más vinculados a lo audiovisual que a la lectura silenciosa se ambientó la narrativa del cuento, en colaboración entre pares, con recursos didácticos sensible a las y los lectores (Bustos, 2016; Bustos, Montenegro, Sandoval y Melo, 2018). Para esta actividad se proyectaron en formato de video imágenes del libro infantil con pequeñas dramatizaciones y musicalización pertinentes, en simultáneo con la narración. Esta práctica de lectura recreativa y divertida captó el interés de las y los participantes que se mostraron entretenidos y muchos pidieron verlo nuevamente.

2. Momento de juegos: a través de actividades lúdicas en la pantalla interactiva, juegos de cartas y canciones se facilitaron recomendaciones para un adecuado lavado de manos y de esta forma, mediante el juego y la motivación audiovisual, se intercambiaron saberes.

3. Momento de cierre: para afianzar los aprendizajes construidos durante todo el proceso, se desarrolló la estrategia trabajo colaborativo, buscando con ello la interpretación e integración de los contenidos trabajados, posibilitando en el estudiantado, la fijación del tema a partir de la lectura reflexiva y el intercambio con sus compañeros (Bustos et al., 2018). El estudiantado trabajó en grupos reducidos, entre cinco y siete participantes, en un collage (Figura 10). La consigna fue dibujar y pegar imágenes y leyendas sobre lo aprendido. Posteriormente las 32 producciones grupales se exhibieron en el patio de la escuela (Figura 11). 


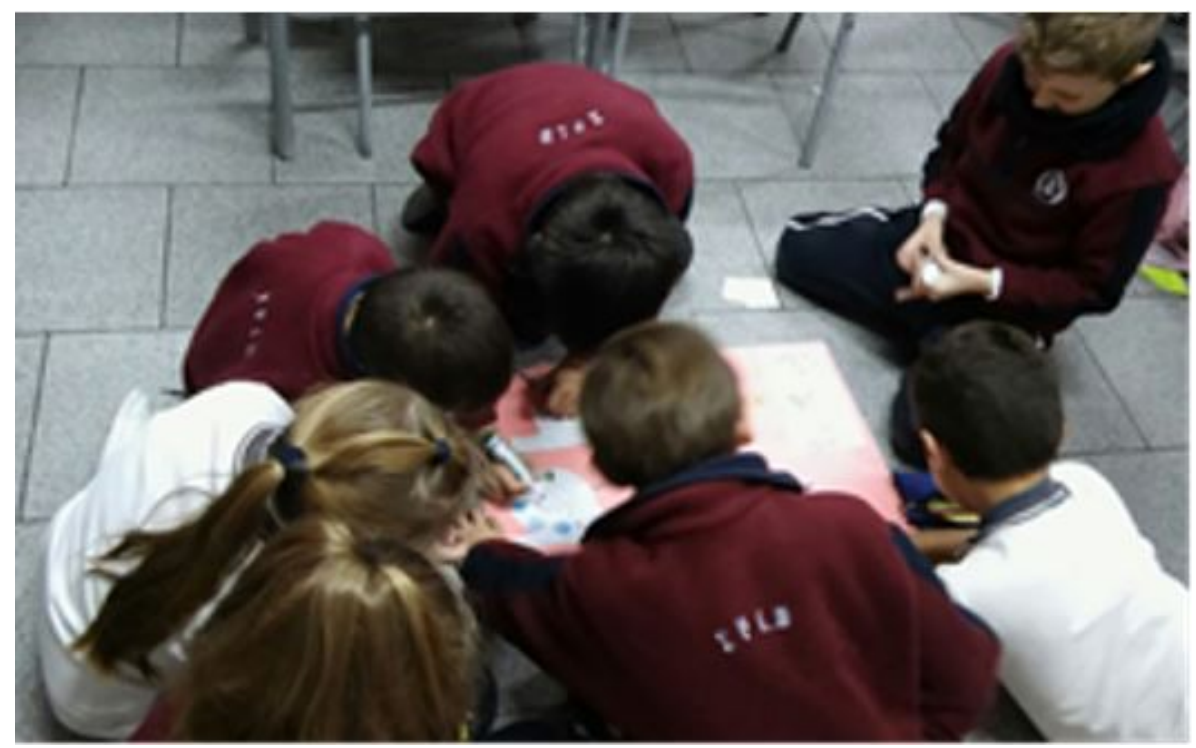

FIGURA 10

Momento de cierre del taller. Fotografía de trabajo en grupo para la producción de collages Fuente: Elaboración propia

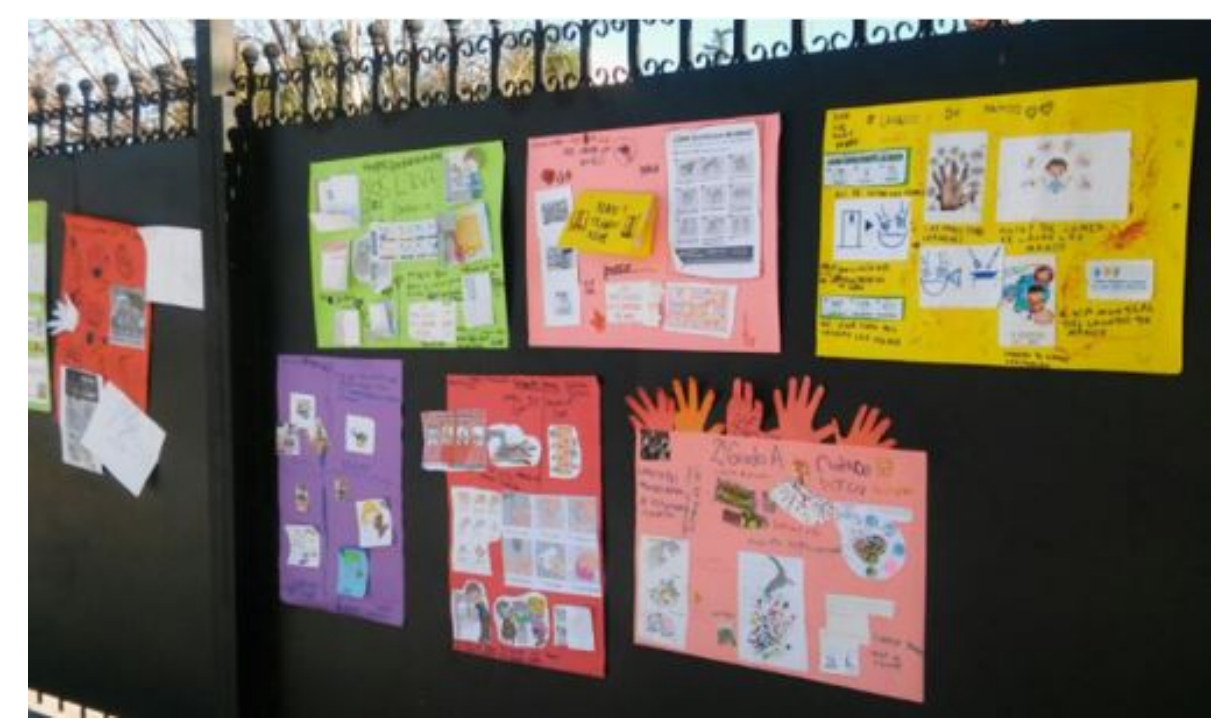

FIGURA 11

Momento de cierre del taller. Fotografía de la exhibición en el patio de la escuela privada de los collages elaborados por el estudiantado

Fuente: Elaboración propia

En el año 2018 en la Noche de los Museos, se presentó una muestra interactiva denominada la higiene de manos en estaciones (Figura 12), el Museo en Ciencias de la Salud del Hospital Nacional de Clínicas, FCMUNC, de la ciudad de Córdoba. Allí, también se exhibieron los 32 collages derivados del trabajo educativo desarrollado en 2017 (Figura 13) con el objeto de visibilizar el Día Mundial del Lavado de Manos y promover el museo y la escuela como espacio de encuentro con la comunidad hospitalaria y el público general, y destacar el rol de la población infantil como promotores de cambios hacia prácticas de higiene correctas. 


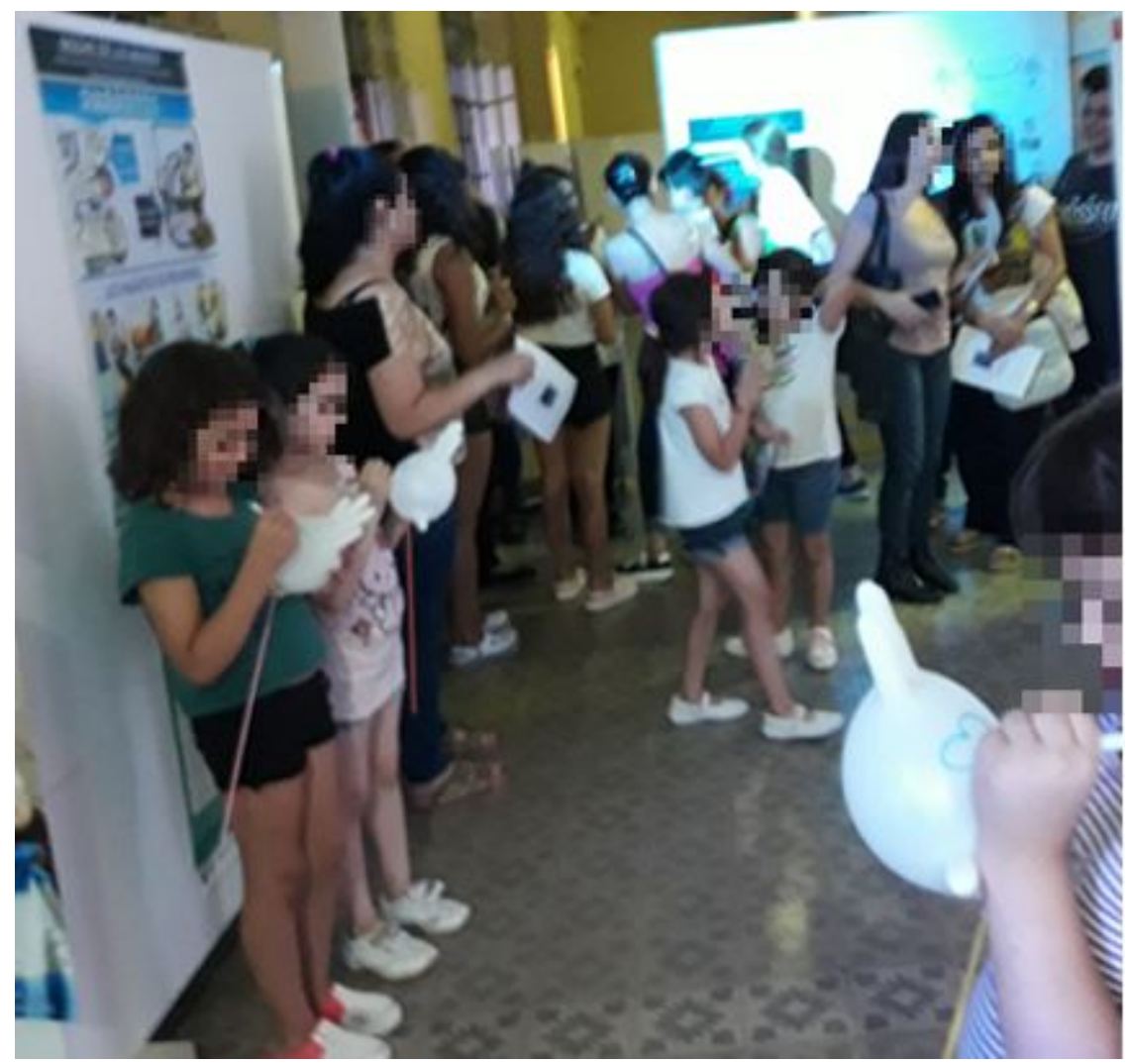

FIGURA 12

Noche de los Museos. Fotografía de la Muestra interactiva

Fuente: Elaboración propia

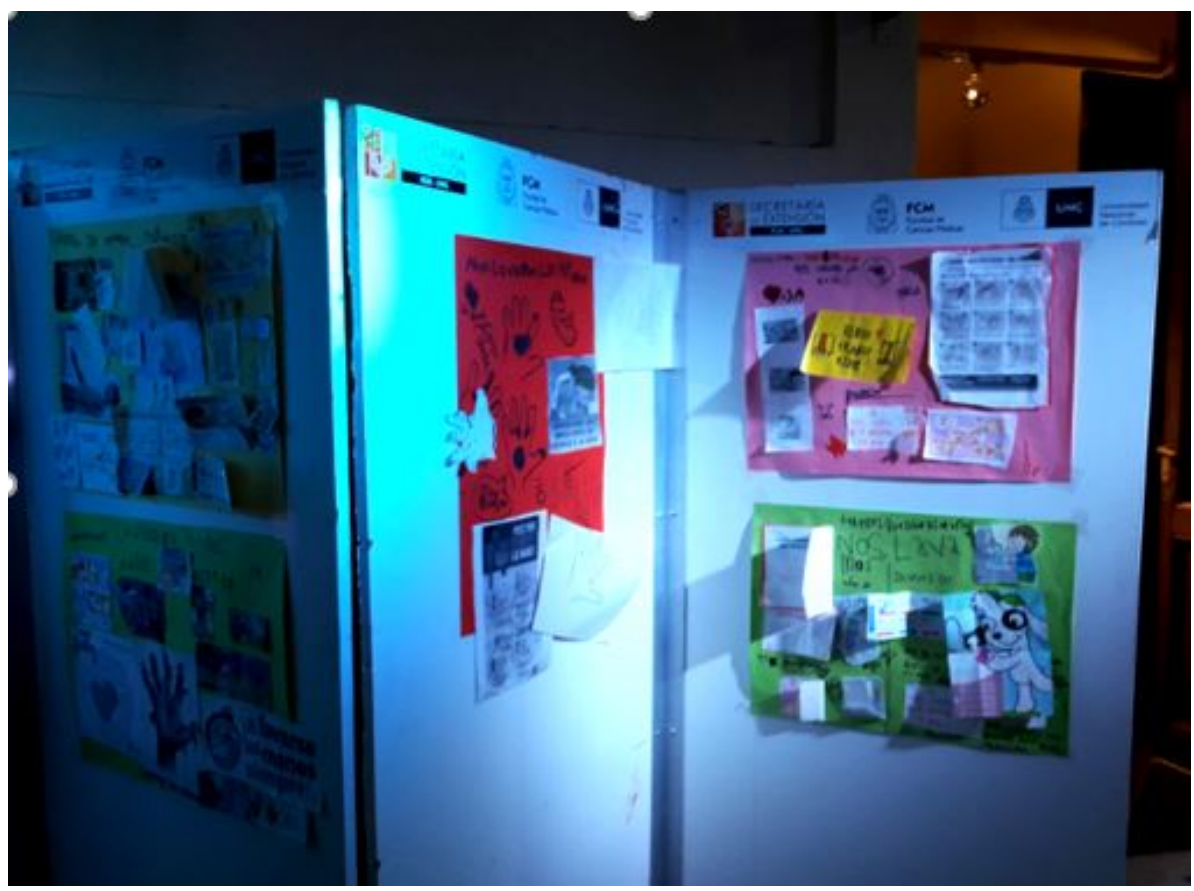

FIGURA 13

Noche de los Museos. Fotografía de la exposición de collages

Fuente: Elaboración propia 
La muestra interactiva fue sectorizada en tres estaciones -juegos, rincón creativo y lavado de manos- para facilitar la ágil participación y minimizar la espera, poniendo en marcha estrategias didácticas divertidas, acordes a la edad de las y los destinatarios. Se desarrollaron dos juegos: el primero consistió en ordenar en un afiche los pasos correctos de lavado de manos y el segundo en reconocer en tarjetas, aquellas imágenes que representan situaciones en las cuales debe higienizar las manos antes de o después de. Para lograr la atención de las y los más pequeños, se trabajó con los colores del semáforo. En el rincón creativo, se obsequiaron globos de manitos que cada participante personalizó con fibrones de colores. La última estación propuso lavarse las manos con alcohol en gel, cantando el feliz cumpleaños como recurso para medir el tiempo de lavado. En esa oportunidad se remplazó la práctica de lavado de manos con agua y jabón por alcohol en gel, debido a no disponer de sanitarios cercanos al stand de la muestra.

\section{Evaluación de la intervención propiamente dicha en la institución educativa y en la noche de los museos}

En el primer año se diseñó una evaluación formativa de conocimientos bajo la modalidad escolar denominada trabajo solito, con la finalidad de recuperar prácticas de higiene de manos y saberes aprendidos en la unidad cuidado del cuerpo impartida por la maestra del aula, como así también los contenidos enseñados por el equipo extensionista. Cada estudiante de la escuela pública (Figura 14) y de la escuela privada (Figuras 15 y 16) pintó, recortó y pegó en su cuaderno ilustraciones con los pasos del lavado de manos con la supervisión del equipo de trabajo. Dicha supervisión consistió en observar, en cada estudiante, como resolvieron la actividad propuesta de ordenar los pasos correctos de lavado de manos, orientando al estudiantado que presentaba dificultades. Finalizada la actividad las docentes a cargo del estudiantado visaron el trabajo solito.

Posteriormente, las maestras completaron una encuesta de satisfacción autoadministrada, individual y anónima para evaluar las actividades desarrolladas en el taller y el libro infantil como herramienta de aprendizaje. Las respuestas a una amplia gama de afirmaciones estuvieron situadas en una escala de valoración del 1 al 5 (1: totalmente en desacuerdo y 5: totalmente de acuerdo).

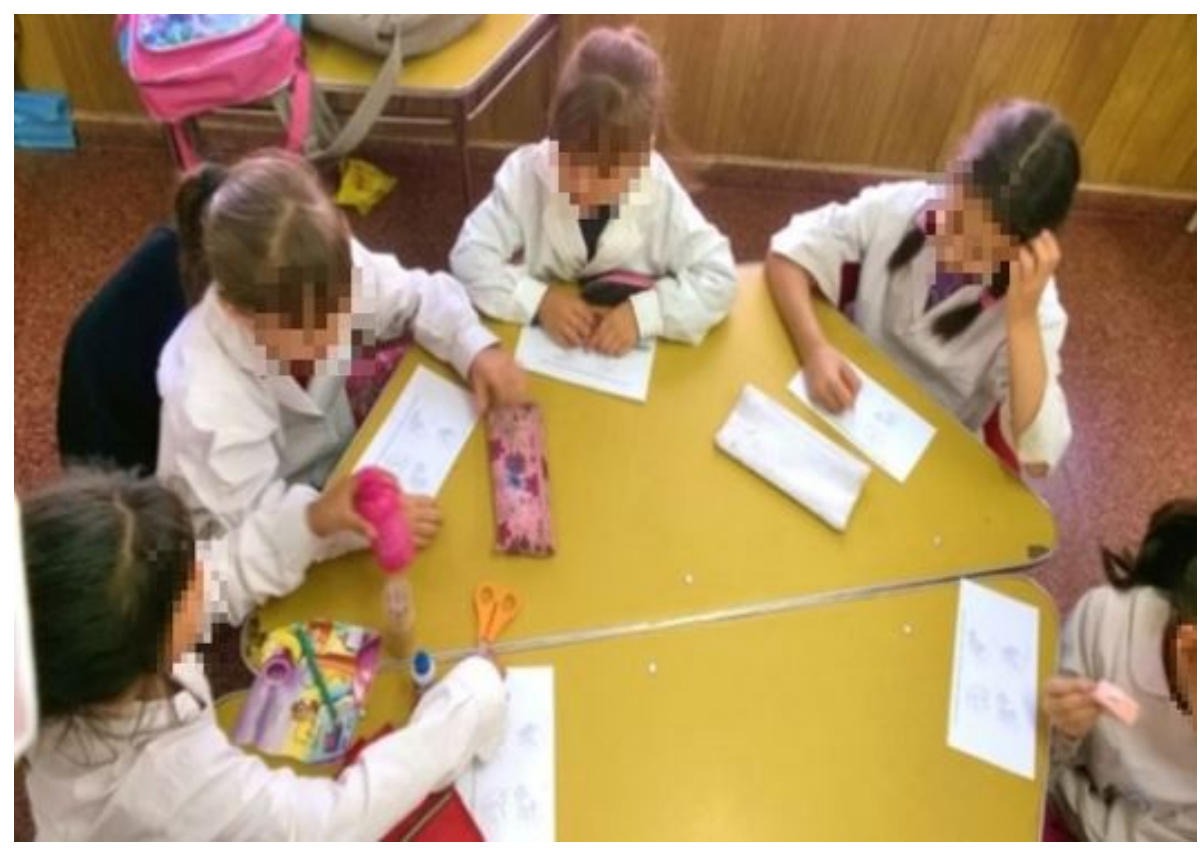

FIGURA 14

Trabajo solito. Fotografía de actividad áulica en la escuela pública

Fuente: Elaboración propia 


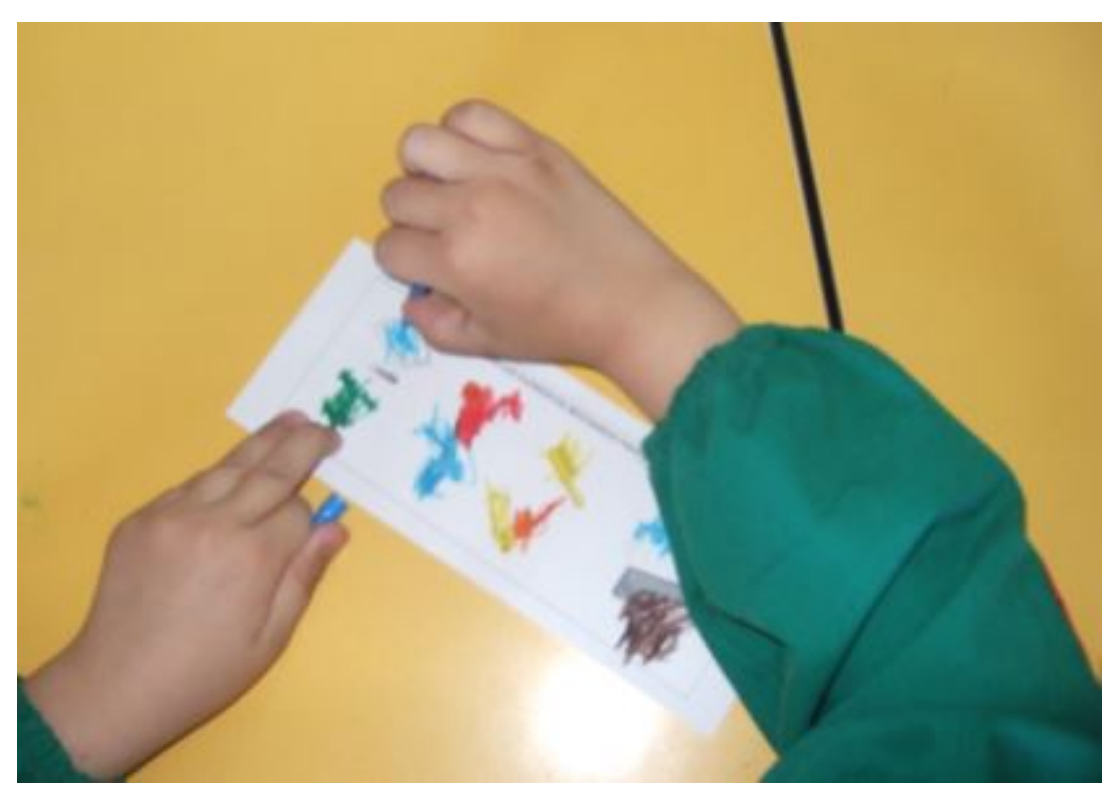

FIGURA 15

Trabajo solito. Fotografía de actividad áulica en la escuela privada

Fuente: Elaboración propia

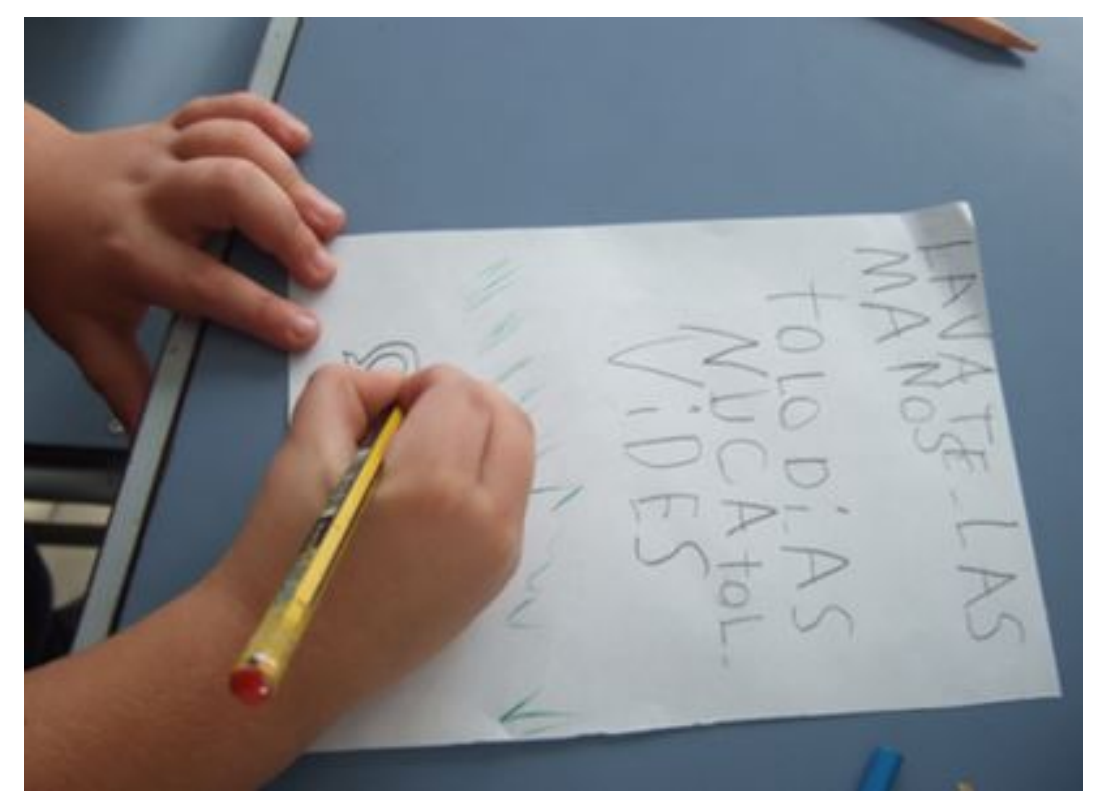

FIGURA 16

Trabajo solito. Fotografía de actividad áulica en la escuela privada

Fuente: Elaboración propia

En el año 2017, se contabilizó la cantidad de estudiantes que se lavaron las manos en los sanitarios y su práctica, teniendo en cuenta los conocimientos enseñados el año anterior. Para ello, durante una jornada escolar, se ejecutó observación directa en los sanitarios con la finalidad de valorar el cumplimiento de los pasos correctos de lavado de manos luego de utilizar el sanitario. El formulario se completó con las opciones: no se lavó, se lavó solo con agua, se lavó con agua y jabón (OMS, 2010). Para la construcción de los collage, se acompañó al estudiantado aportando la información necesaria para el armado adecuado de los mismos.

Con la finalidad de evaluar las actividades, las maestras completaron una encuesta de satisfacción autoadministrada, individual y anónima con una escala de valoración del 1 al 5 (1: totalmente en desacuerdo y 5: totalmente de acuerdo). 
En el año 2018, al finalizar la exposición en el Museo, estudiantes y docentes universitarios ejecutaron una evaluación cualitativa, indagando a cada participante, ¿qué opina sobre la experiencia? a fin de analizar la actividad y generar nuevas alternativas de abordaje para futuras intervenciones.

\section{Producción de materiales educativos}

Las producciones derivadas de la intervención en las escuelas, durante el primer año de trabajo, fueron dos, afiches de difusión y el libro infantil:

Afiches de difusión: se diseñaron varios modelos de carteles tamaño A3 con ilustraciones y mensajes educativos. Estos se elaboraron con base en las recomendaciones de la OPS (2011), junto a la información recabada en las escuelas.

Libro infantil: Para su diseño se observaron las prácticas de higiene de niñas y niños con una guía de observación en los sanitarios y talleres que completaron las y los estudiantes universitarios y se rescataron los dibujos y narraciones de las vivencias en el trabajo en las escuelas. Se tuvieron en cuenta los ejes conceptual, pedagógico y comunicacional, para adecuar los contenidos a la población destinataria.

\section{Resultados}

Durante los meses de septiembre y octubre del año 2016 participaron en las actividades un total de 316 alumnos provenientes de nivel inicial (salas de 4 y 5 años) y de nivel primario $\left(1^{\circ}\right.$ y $2^{\circ}$ grado) de las escuelas privada (Tabla 1$)$ y pública (Tabla 2 ).

Tabla 1

Estudiantes participantes en la escuela privada, año 2016

TABLA 1

Estudiantes participantes en la escuela privada, año 2016

\begin{tabular}{|c|c|c|c|c|}
\hline \multicolumn{2}{|c|}{ ESCUEL_A PRIVADA } & \multirow{2}{*}{$\begin{array}{l}\text { NIÑAS } \\
37\end{array}$} & \multirow{2}{*}{$\begin{array}{l}\text { NIÑOS } \\
32\end{array}$} & \multirow{3}{*}{$\begin{array}{l}\text { TOTAL } \\
69 \\
33 \\
\end{array}$} \\
\hline & $1^{\circ}$ Grado & & & \\
\hline PRIMARIA & $2^{\circ}$ Grado & 17 & 16 & \\
\hline \multirow{3}{*}{$\begin{array}{l}\text { JARDÍN Sala de } \\
4\end{array}$} & Sala violeta & 14 & 11 & \multirow{3}{*}{73} \\
\hline & Sala naranja & 16 & 9 & \\
\hline & Sala turquesa & 11 & 12 & \\
\hline \multirow{2}{*}{$\begin{array}{l}\text { JARDÍN Sala de } \\
5\end{array}$} & Sala roja & 22 & 11 & \multirow{2}{*}{69} \\
\hline & Sala azul & 19 & 17 & \\
\hline \multicolumn{4}{|c|}{ TOTAL DE ESTUDIANTES PARTICIPANTES } & 244 \\
\hline
\end{tabular}

Fuente: Elaboración propia 
TABLA 2

Estudiantes participantes en la escuela pública, año 2016

\begin{tabular}{lllll}
\hline ESCUELA PÚBLICA & NIÑAS & NIÑOS & TOTAL \\
\hline $\begin{array}{l}\text { PRIMARIA Turno } \\
\text { mañana }\end{array}$ & $1^{\circ}$ Grado & 9 & 8 & 17 \\
& $2^{\circ}$ Grado & 3 & 6 & 9 \\
\hline $\begin{array}{l}\text { PRIMARIA Turno } \\
\text { tarde }\end{array}$ & $1^{\circ}$ Grado & 10 & 7 & 17 \\
\hline $\begin{array}{l}\text { JARDÍN Sala de } \\
5\end{array}$ & $\begin{array}{l}\text { Turno mañana } \\
\text { Turno tarde }\end{array}$ & 3 & 8 & 29 \\
\hline \multicolumn{7}{l}{ TOTAL DE ESTUDIANTES PARTICIPANTES } \\
\hline
\end{tabular}

Fuente: Elaboración propia

Se observó los sanitarios de ambas escuelas y, si bien se encontraron limpios y en condiciones edilicias adecuadas, ninguno contó con jabón líquido y toalla descartable. Sin embargo, el estudiantado de nivel inicial, disponía de toalla y jabón en barra individual al tener pautado el lavado de manos previo a la merienda. A su vez, se evidenció que uno de cada diez alumnos que ingresaron a los sanitarios se lavó las manos solo con agua. Se obsequiaron 4 kit de jabón líquido y papel descartable para cada escuela.

En la organización de los talleres, en ambas escuelas, si bien existieron momentos de dispersión con los más pequeños, ningún estudiante mostró disconformidad en efectuar las actividades, ni surgieron problemas de comportamiento. Se contó con la colaboración de las maestras en la organización, orden y pautas durante el trabajo y si bien ambas Instituciones educativas se comprometieron en incluir dentro del presupuesto anual, jabón líquido y papel descartable en los sanitarios, dichos insumos se suministraron de forma discontinua.

En general, el estudiantado no tuvo inconvenientes al escribir las consignas del trabajo solito, ordenando correctamente la secuencia de lavado de manos. Al finalizar la intervención en 2016, la encuesta de satisfacción fue respondida por nueve de las trece maestras debido a que, tres docentes se encontraban con carpeta médica y una con licencia por maternidad. En relación con el trabajo que se llevó a cabo en las escuelas, la mayoría estuvo totalmente de acuerdo en que fue una experiencia interesante, con actividades claras, participativas y relacionadas con el currículo escolar. El 88\% estuvo totalmente de acuerdo en que el libro motiva a seguir trabajando en la temática y el 100\% totalmente de acuerdo en que es un material ilustrativo adecuado al nivel lingüístico y de conocimientos de los destinatarios. Destacando la organización, secuenciación y formato.

En cuanto a los afiches de difusión elaborados se obsequiaron 15 copias para colgar en las aulas de cada escuela (Figuras 17 y 18). 


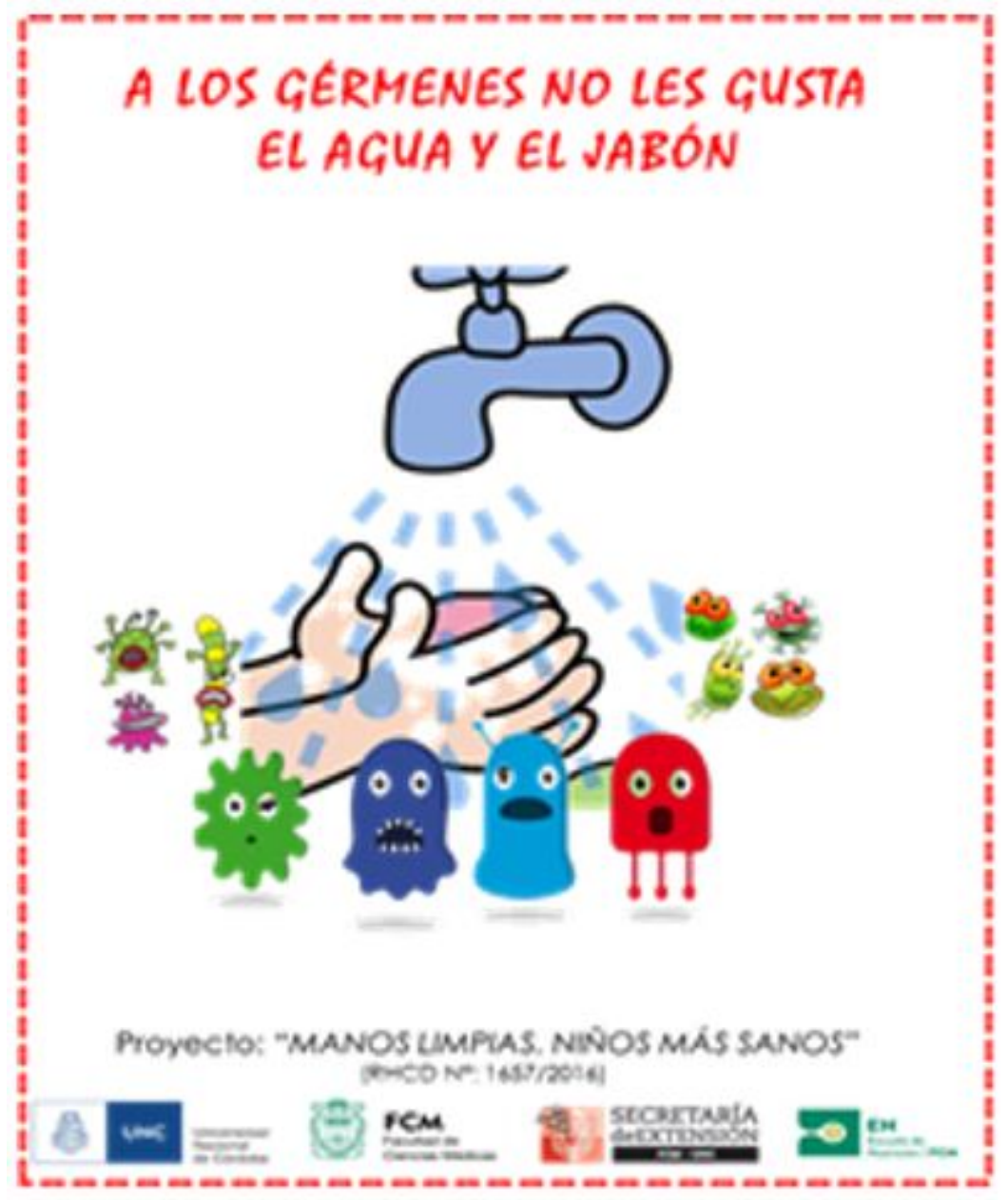

FIGURA 17

Afiche de difusión. Promoción del lavado de manos Fuente: Elaboración propia en base a OPS (2011) 


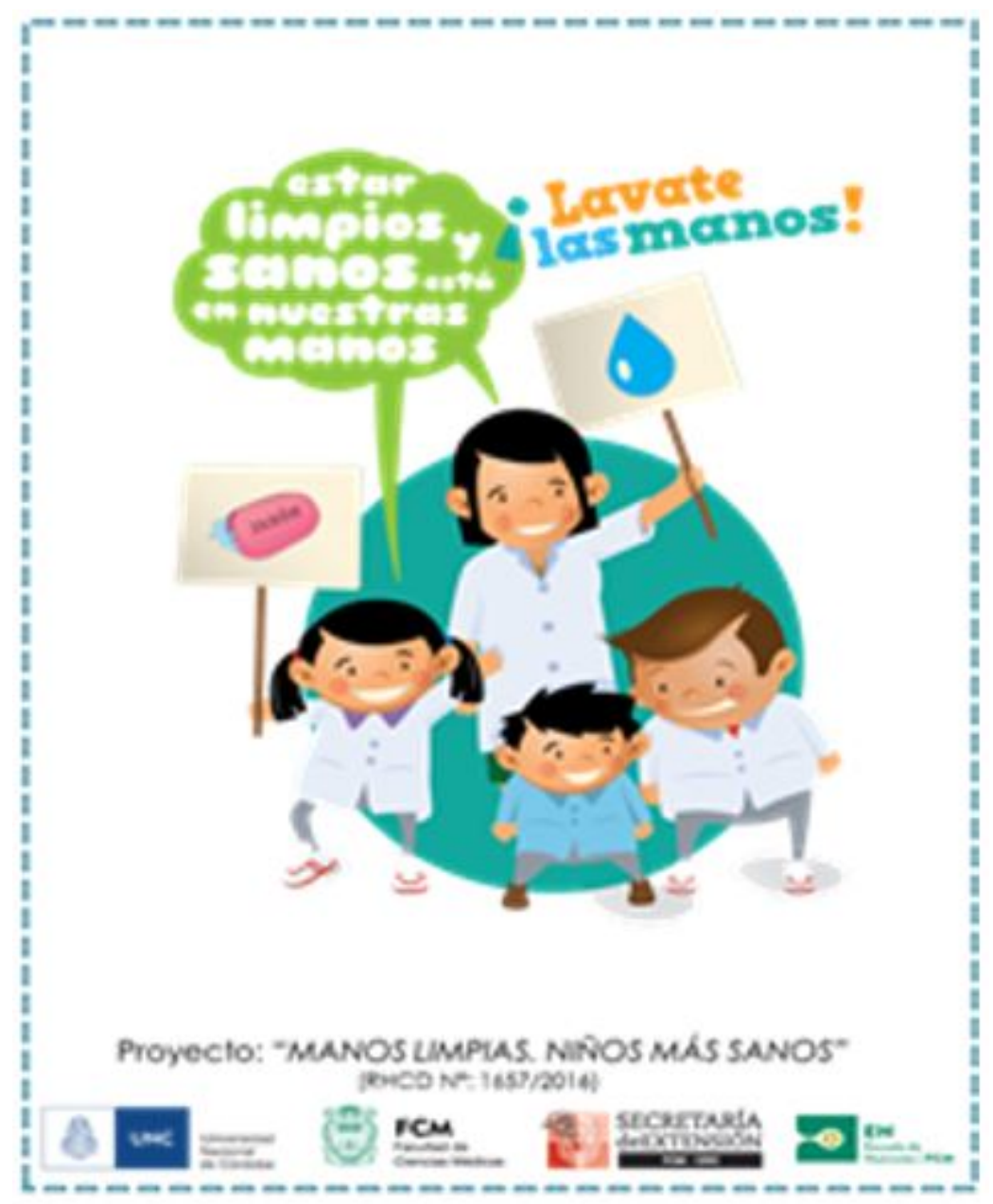

FIGURA 18

Afiche de difusión. Promoción del lavado de manos

Fuente: Elaboración propia en base a OPS (2011)

Las acciones del presente trabajo participativo dieron como resultado la redacción, ilustración y publicación de un libro infantil Manos limpias, niños más sanos (Oberto et al., 2016), con los pasos correctos del lavado de manos. En las figuras 19 y 20 se presenta la portada y algunas páginas del libro infantil elaborado, a modo de ejemplo. Se entregaron dos ejemplares para la biblioteca de cada sala y grado participantes, con la finalidad de que permanezcan como material de lectura complementaria en el desarrollo de los contenidos relacionados a la salud y al cuidado del cuerpo. Con el apoyo de la Secretaría de Extensión Universitaria, FCM-UNC, se editaron 220 ejemplares con la intención de continuar trabajando con el material en otras escuelas. 


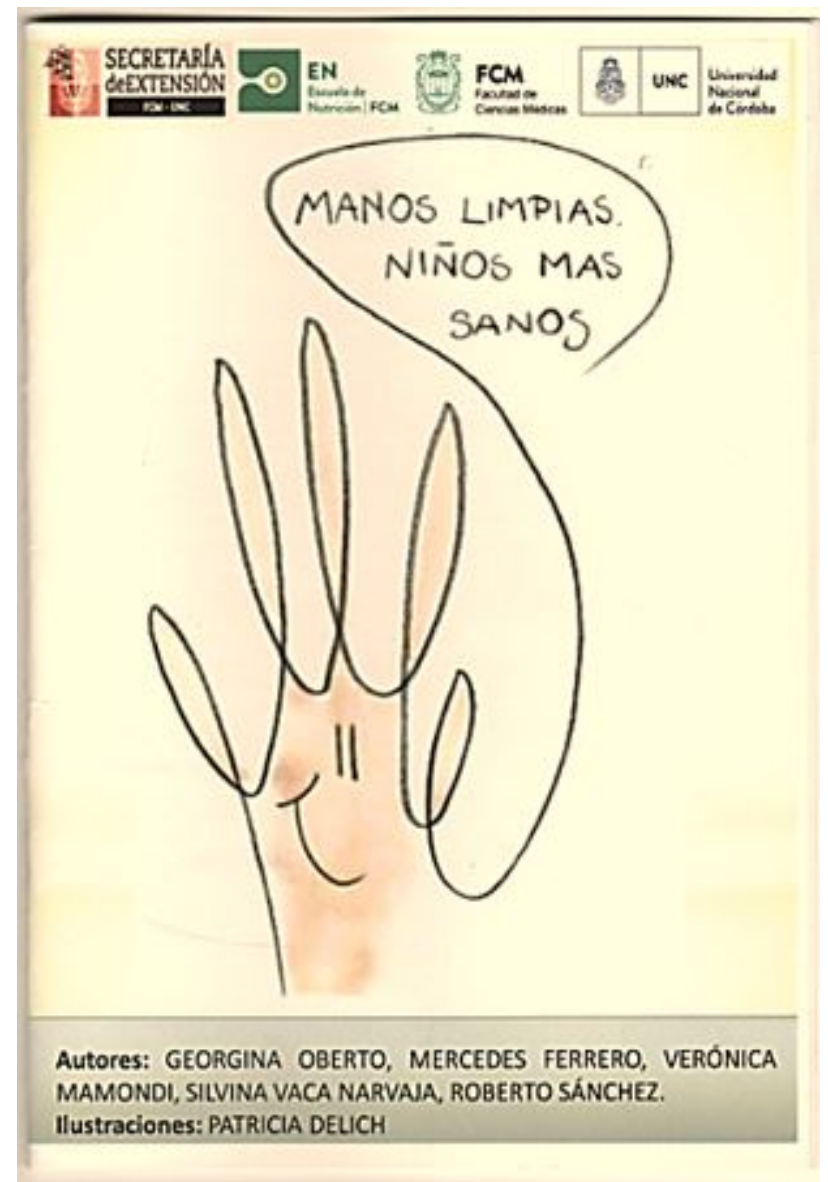

\section{FIGURA 19}

Portada. Libro infantil: Manos limpias. Niños más sanos Fuente: Oberto et al., 2016.

"QUÉ HACÉS, TOM!!

UY...LULI, ME ASUSTATE ¿QUÉ PASA? - ¡NO PODÉS PONERTE A COMER CON ESAS MANOS TAN SUCIAS! ACABÁS DE JUGAR A LA PELOTA...

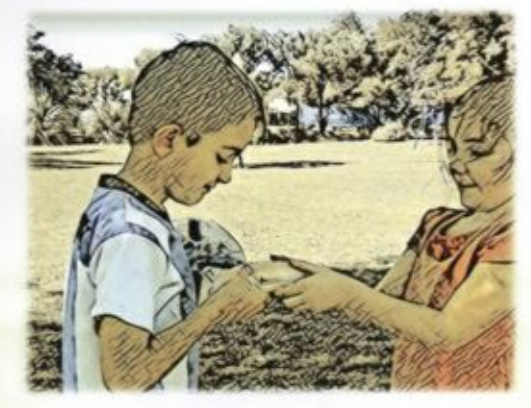

- ¿Y QUE IMPORTA QUE TENGA LAS MANOS SUCIAS? NO PASA NADA...

- ¿QUÉ DECIS TOMI! ¡NO SABÉS QUE EN LAS MANOS SUCIAS ESTAN LOS GÉRMENES? - ¿ESTAN LOS QUÉEEEE? ¿QUÉ SON LOS
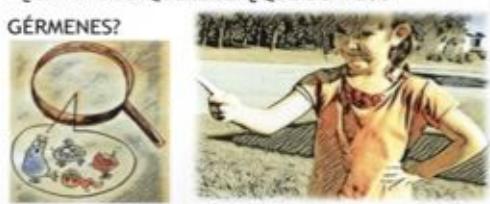

-LOS GÉRMENES, TOMI, SON COMO BICHITOS MUY CHIQUITITOS, QUE NO LOS PODEMOS VER, PERO SON LOS QUE CAUSAN LAS ENFERMEDADES. - ¿ENTONCES SI LOS TENGO EN MIS MANOS ME PUEDO ENFERMAR, LULI?
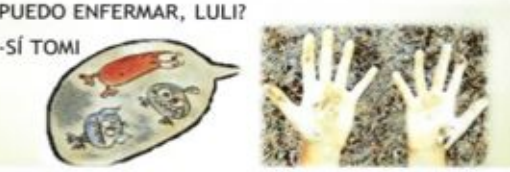

FIGURA 20

Páginas del contenido. Libro infantil: Manos limpias. Niños más sanos Fuente: Oberto et al., 2016 
En el año 2017, en la escuela privada, participaron 188 niños y niñas de $1^{\circ}, 2^{\circ}$ y $3^{\circ}$ grado (Tabla 3 ). Cabe aclarar que la mayoría de esos estudiantes había participado en el año 2016.

TABLA 3

Estudiantes participantes en la escuela privada, año 2017

\begin{tabular}{lllll}
\hline ESCUELA PRIVADA & NIÑAS & NIÑOS & TOTAL \\
\hline PRIMARIA & $\begin{array}{l}1^{\circ} \text { Grado }^{\circ} \\
\text { Grado } 3^{\circ}\end{array}$ & 351935 & 343431 & 695366 \\
\hline TOTAL DE ESTUDIANTES PARTICIPANTES & & $\mathbf{1 8 8}$ \\
\hline
\end{tabular}

Fuente: Elaboración propia

Se puede observar que los sanitarios se encontraron limpios y luego del primer recreo el personal de limpieza lo aseó. Además, se registró durante los dos recreos de una jornada educativa, cuántos estudiantes se lavaron las manos después de utilizar los sanitarios. Se contabilizaron 40 niñas lavándose las manos, de las cuales 7 la efectuaron incorrectamente, puesto que solo mojaron sus manos con agua o directamente no se lavaron.

Por otro lado, de 41 niños y niñas que ingresaron a los sanitarios, se evidenció la práctica correcta de lavado de manos en 14 de ellos, y de 27 restantes, solo 10 se mojaron con agua y el resto no se lavó las manos. Si bien la práctica de lavado de manos continuó siendo baja, había mejorado notablemente con respecto al año anterior.

En el año 2017, siete maestras respondieron la encuesta (seis a cargo de los grados participantes y una maestra integradora de $2^{\circ}$ grado A). El $100 \%$ de las encuestadas estuvo totalmente de acuerdo con el alto grado de participación del estudiantado en el juego y que este podría afianzar la apropiación de conocimientos en sus alumnos. El 86\% consideró que las actividades lúdicas se explicaron de forma ordenada y clara. El 100\% estuvo totalmente de acuerdo en que la actividad grupal fue pertinente a cada destinatario. El 86\% estuvo totalmente de acuerdo en que los collages podrían actuar como disparador para el resto del estudiantado de la escuela.

En el año 2018 participaron de la muestra en el Museo alrededor de 60 niños y niñas entre 3 y 10 años acompañados por sus familias. Ningún participante mostró disconformidad en desarrollar las actividades, ni surgieron problemas de comportamiento.

Las personas cuidadoras alentaron la participación e interacción, permitiendo al equipo de trabajo establecer un vínculo con la población infantil y su entorno, favoreciendo la construcción colectiva de conocimientos y la retroalimentación de saberes. Algunas de las respuestas obtenidas en la evaluación cualitativa fueron las siguientes: "me resultó una experiencia enriquecedora por el contacto con los niños"(Participante 1, comunicación personal, 26 de octubre de 2018); "si bien existen barreras como la timidez de algunos niños, los juegos los entusiasma a participar" (Participante 2, comunicación personal, 26 de octubre de 2018); "para mi sorpresa la demanda de la población fue mayor de lo esperado"(Participante 3, comunicación personal, 26 de octubre de 2018); "siento que son espacios muy gratificantes" (Participante 4, comunicación personal, 26 de octubre de 2018).

\section{DiSCUSIÓN Y CONCLUSIONES}

El desarrollo de este trabajo de educación para la salud se enmarca dentro de la Universidad como extensión de su labor hacia la sociedad (Pérez, Farah y Rulli, 2018) con la finalidad de contribuir a promover en las escuelas el correcto lavado de manos como método de prevención de ETA y de otras enfermedades diarreicas y respiratorias. Se considera que la intervención podría favorecer un cambio de conducta en los 
hábitos higiénicos cotidianos de la población infantil y maestras de las Instituciones educativas destinatarias. Si bien el estudiantado tuvo la experiencia de lavarse las manos como parte del proceso que vincula el aprendizaje-reflexión-acción, la práctica de esta conducta higiénica continuó siendo insuficiente en el tiempo evaluado. Revisiones sistemáticas concluyen que es muy heterogénea la evidencia sobre los cambios en los comportamientos o mejoras en los conocimientos en escolares sobre la higiene de manos, se requiere realizar más investigaciones y evaluaciones de las intervenciones (Mbakaya, Lee y Lee, 2017; McMichael, 2019).

Además, se pudo constatar que no se mantuvo el suministro permanente en los sanitarios de los elementos higiénicos necesarios para la práctica correcta del lavado de manos (jabón líquido y toallas descartables). Existe amplia evidencia de que la infraestructura y los recursos para la higiene de manos son importantes para promover y sostener los comportamientos de higiene y la reducción del riesgo de enfermedades relacionadas con el lavado de manos (McMichael, 2019). Por otro lado, el acompañamiento de las maestras en esta práctica, fue dispar. En el nivel inicial, esta tarea forma parte de las actividades habituales de las maestras que acompañaron a las y los infantes frecuentemente en el lavado de manos. En tanto que, en el nivel primario, se observó la colaboración de las maestras en el lavado de manos de las y los alumnos, durante la presente intervención, en el desarrollo de los talleres; sin embargo, esta práctica de acompañamiento en los sanitarios, no se mantuvo en forma cotidiana, diariamente, argumentando que es escaso el personal para acompañar a tantos alumnos.

En cuanto al libro infantil, se estima que es un material didáctico ilustrativo con lenguaje sencillo para el entendimiento e interés del estudiantado, por lo tanto podría representar una contribución importante para la educación para la salud en las escuelas. Integrando sus bibliotecas, serviría como material de lectura complementaria en el abordaje de los contenidos relacionados a la salud y el cuidado del cuerpo. El libro impreso constituye el material didáctico de uso general en la mayoría de las escuelas argentinas y algunos autores ponderan -una de sus ventajas más importantes- la circulación libre y pública de información (Caputi, 2014).

La intervención de la Universidad en esta experiencia de educación para la salud escolar, dando cuenta de su responsabilidad social, permitió la participación del estudiantado y lo involucró activamente en el proceso de aprendizaje de las correctas prácticas higiénicas-sanitarias. Todas las estrategias puestas en marcha durante el trabajo, como las exposiciones dialogadas, los talleres y las propuestas lúdicas, motivaron a las y los alumnos para la producción de material ilustrativo. Siguiendo estrategias pedagógicas desde un enfoque constructivista, el estudiantado puede construir sus ideas, conocimiento y experiencias en el marco de un proceso de construcción o reconstrucción de la realidad en interacción entre las personas y el mundo (Martínez y Zea, 2004). Así, las y los estudiantes se convirtieron en protagonistas fundamentales de la educación para la salud, actuando como promotores de hábitos saludables y claros difusores de acciones preventivas entre sus pares con sus producciones finales (afiches, mensajes educativos, libro infantil y collages).

Hay personas autoras que han demostrado el impacto positivo de las intervenciones educativas para la promoción de la salud (Caballero et al, 2017). La interacción de las comunidades educativas, nivel universitario, inicial y primario, permitió un intercambio de saberes enriqueciendo la práctica educativa. De esta forma, los actores universitarios facilitaron conocimientos en relación con la educación para la salud, y a la vez, recibieron de las escuelas pública y privada sus experiencias curriculares respecto de las prácticas relacionadas al cuidado de la salud. Estos saberes mostraron una firme vinculación, no solo con los currículos, sino también con el contexto socio-económico de cada escuela. Por lo tanto, fue imprescindible planificar las actividades para cada Institución en particular, que estuvieran acordes con el perfil de su comunidad educativa. La escuela es uno de los ámbitos ideales para las intervenciones de promoción de la salud, no solo por el hecho de concentrar una gran cantidad de personas, sino también porque al integrar en su currículo contenidos de educación para salud, se pueden articular estas intervenciones (Rodríguez et al, 2017). 
En definitiva, el aporte disciplinar y pedagógico de la intervención, permitió establecer un vínculo con el estudiantado fortaleciendo la retroalimentación de saberes y la construcción colectiva de conocimientos, dando cuenta de una de las misiones de la Universidad, sobre la extensión y vinculación comunitaria (REXUNI, 2012). Además, permitió crear un entorno favorable para promover comportamientos saludables, sin embargo, en concordancia con las conclusiones de otros autores en cuanto al efecto de la intervención educativa en los resultados del lavado de manos de escolares (Wichaidit et al,2019), se considera necesario continuar fortaleciendo las prácticas con futuras intervenciones e investigaciones que evalúen el impacto de las mismas.

\section{REFERENCIAS}

Beltrán, J., Navarro, B. y Peña, S. (2018). Prácticas que obstaculizan los procesos de transposición didáctica en escuelas asentadas en contextos vulnerables: Desafíos para una transposición didáctica contextualizada. Revista Educación, 42(2), 335-355. doi: https://doi.org/10.15517/revedu.v42i2.27571

Bustos, I.A., Montenegro V.C., Sandoval, W. y Melo, G. (2018). Creando contextos de comprensión: Rol estudiantil en las instancias de lectura en la sala de clases. Revista Educación, 42(2), 1-30. doi: https://doi.org/10.15517/r evedu.v42i2.23432

Bustos, S.G. (2016). Lector no se nace, se hace. Sociales y Virtuales. Recuperado de http://socialesyvirtuales.web.unq .edu.ar/articulos/lector-no-se-nace-se-hace/

Caballero, C.R., Flores, J.F., Bonilla, P. y Arenas, L. (2017). Experiencias de promoción de la salud en escuelas de nivel primario en México. Revista Memorias del Instituto de Investigaciones en Ciencias de la Salud, 15(1), 22-32. doi: https://doi.org/10.18004/Mem.iics/1812-9528/2017.015(01)22-032

Caputi, D.C. (2014). El libro de texto: una mirada más. Sociales y Virtuales. Recuperado de: http://socialesyvirtuales .web.unq.edu.ar/articulos-de-los-estudiantes/el-libro-de-texto/

Ejemot, R.I., Ehiri, J.E., Arikpo, D., Meremikwu, M.M. y Critchley J.A. (2015). Hand washing promotion for preventing diarrhoea. Cochrane Database of Systematic Reviews, 3(9). doi: https://doi.org/10.1002/14651858. CD004265.pub3

Fondo de las Naciones Unidas para la Infancia [UNICEF]. (2018). Infografia: Algunos datos sobre el lavado de manos. Lavarse las manos con jabón es una de las maneras más fáciles de mantener sanos a los niños. Recuperado de: http s://www.unicef.org/es/historias/infografia-algunos-datos-sobre-el-lavado-de-manos

Global Handwashing Partnership (2017). Día Mundial del Lavado de Manos. Guía para planificadores.

Haverstick, S., Goodrich, C., Freeman, R., James, S., Kullar, R. y Ahrens, M. (2017). Patients' Hand Washing and Reducing Hospital-Acquired Infection. Critical Care Nurse, 37(3), 1-8. doi: https://doi.org/10.4037/ccn201 7694.

Herrero, S., López, J. y Martínez, J. (2017). Entornos saludables escolares. ¿Por qué no tiene éxito la generación de entornos saludables en la escuela? Revista Iberoamericana de Enfermeria Comunitaria, 10(2), 21-29.

Ippolito, J. y Mantilla, L. (2003). Fortalecimiento de la Iniciativa Regional Escuelas Promotoras de la Salud: Estrategias y Lineas de Acción 2003-2012. Washington, D.C.: OPS.

Luby, S.P., Halder, A.K., Huda, T., Unicomb, L. y Johnston, R.B. (2011) The Effect of Handwashing at Recommended Times with Water Alone and With Soap on Child Diarrhea in Rural Bangladesh: An Observational Study. PLOS Medicine, 8(6), 1-12. doi: https://doi.org/10.1371/journal.pmed.1001052

Martínez, E. y Zea, E. (2004). Estrategias de enseñanza basadas en un enfoque constructivista. Revista Ciencias de la Educación, 4(2), 69-90.

Mbakaya, B.C., Lee, P.H. y Lee, R.L. (2017). Hand Hygiene Intervention Strategies to Reduce Diarrhoea and Respiratory Infections among Schoolchildren in Developing Countries: A Systematic Review. Review International journal of environmental research and public health, 14(4), 1-14. doi: https://doi.org/10.3390/ij erph 14040371 
McMichael, C. (2019). Water, Sanitation and Hygiene (WASH) in Schools in Low-Income Countries: A Review of Evidence of Impact. Review International journal of environmental research and public health, 16(3), 1-21. doi: https://doi.org/10.3390/ijerph16030359

Morón, J.A. (2015). Investigar e intervenir en educación para la salud. Madrid, España: Narcea.

Oberto, M.G., Ferrero, M., Mamondi, V., Vaca, S. y Sánchez R. (2016). Manos limpias, niños más sanos. Córdoba, Argentina: Editorial Copy-Rápido.

Organización Mundial de la Salud [OMS] (1986). Carta de Ottawa para la promoción de la Salud. Primera Conferencia Internacional sobre la Promoción de la Salud. Ottawa, Canadá. Recuperado de https://bit.ly/2urXmff

Organización Mundial de la Salud [OMS] (2010).¿Cómo lavarse las manos? Recuperado de https://bit.ly/39TL9QM

Organización Mundial de la Salud [OMS] (2016). Novena Conferencia Mundial de Promoción de la Salud. Consenso de Shanghai sobre Ciudades Saludables. Recuperado de https://bit.ly/35DqexY

Organización Panamericana de la Salud [OPS] (2011). Día Mundial del Lavado de Manos. Recuperado de https:// bit.ly/36NVtI0

Pérez, C., Farah, E. y Rulli, F. (2018). Cómo luchar contra microorganismos resistentes a medicamentos. Difusión de conocimientos científicos en una escuela secundaria. Revista Educación. 42(2). doi: https://doi.org/10.15517/ revedu.v42i2.24246

Red Nacional de Extensión Universitaria [REXUNI]. (2012). Plan Estratégico 2012-2015 de la REXUNI en el Acuerdo Plenario No 811/12. Colombia: Consejo Interuniversitario Nacional.

Resolución No 211. (2017). Honorable Consejo Directivo, Facultad de Ciencias Médicas, Universidad Nacional de Córdoba, del 22 de marzo de 2017. Boletín 9 (8), 50-50. Recuperado de https://bit.ly/2uz7gfr

Resolución No 1450. (2017). Honorable Consejo Directivo, Facultad de Ciencias Médicas, Universidad Nacional de Córdoba, del 23 de agosto de 2017. Boletín 32 (8), 26-26. Recuperado de https://bit.ly/2QVaiC2

Resolución No 859. (2018). Honorable Consejo Directivo, Facultad de Ciencias Médicas, Universidad Nacional de Córdoba, del 11 de diciembre de 2018. Boletin 3 (10), 24-24. Recuperado de https://bit.ly/36BCWPp

Rodríguez, A.F., Páez, R.E., Altamirano, E.J., Paguay, F.W., Rodríguez, J.C. y Calero, S. (2017). Nuevas perspectivas educativas orientadas a la promoción de la salud. Educación Médica Superior, 32(4), 1-14.

Soto, V.I., Gómez, O.J., Parrado, Y.M., Hernández, P. y Gómez, A.P. (2017). Caracterización de hábitos de higiene y ambientes en lugares de atención integral a población infantil. Revista da Escola de Enfermagem da USP, 51:1-7. doi: http://dx.doi.org/10.1590/S1980-220X2016042103264

Universidad Nacional de Córdoba [UNC] (2018). Noche de los Museos 2018. Recuperado de https://bit.ly/35xnPVP

Wichaidit, W., Steinacher, R., Okal, J.A., Whinnery, J., Null, C., Kordas, K. y Ram, P.K. (2019). Effect of an equipment-behavior change intervention on handwashing behavior among primary school children in Kenya: the Povu Poa school pilot study. BMC public health, 19(1), 1-12. doi: https://doi.org/10.1186/s12889-019-6 902-2

Willmott, M., Nicholson, A., Busse, H., MacArthur, G.J., Brookes, S. y Campbell, R. (2015). Effectiveness of hand hygiene interventions in reducing illness absence among children in educational settings: A systematic review and meta-analysis. Archives of in Childhood, 101(1), 42-50. doi: https://doi.org/10.1136/archdischild-2015-3 08875

\section{BY-NC-ND}

